OPEN ACCESS

Edited by:

Yan Xu,

Third Affiliated Hospital of Sun Yat-sen

University, China

Reviewed by:

Björn Rath,

Klinikum Wels-Grieskirchen, Austria

Yuelin Zhang,

Department of Emergency and Critical

Care Medicine, Guangdong Academy

of Medical Sciences, China

*Correspondence:

James Adjaye

James.Adjaye@med.uni-

duesseldorf.de

Specialty section:

This article was submitted to

Stem Cell Research,

a section of the journal

Frontiers in Cell and Developmental

Biology

Received: 31 May 2021

Accepted: 17 August 2021

Published: 16 September 2021

Citation:

Wruck W, Graffmann N, Spitzhorn L-S and Adjaye J (2021)

Human Induced Pluripotent Stem Cell-Derived Mesenchymal Stem Cells Acquire Rejuvenation and Reduced

Heterogeneity.

Front. Cell Dev. Biol. 9:717772.

doi: 10.3389/fcell.2021.717772

\section{Human Induced Pluripotent Stem Cell-Derived Mesenchymal Stem Cells Acquire Rejuvenation and Reduced Heterogeneity}

\author{
Wasco Wruck, Nina Graffmann, Lucas-Sebastian Spitzhorn and James Adjaye* \\ Medical Faculty, Institute for Stem Cell Research and Regenerative Medicine, Heinrich Heine University Düsseldorf, \\ Düsseldorf, Germany
}

Despite the uniform selection criteria for the isolation of human mesenchymal stem cells (MSCs), considerable heterogeneity exists which reflects the distinct tissue origins and differences between individuals with respect to their genetic background and age. This heterogeneity is manifested by the variabilities seen in the transcriptomes, proteomes, secretomes, and epigenomes of tissue-specific MSCs. Here, we review literature on different aspects of MSC heterogeneity including the role of epigenetics and the impact of MSC heterogeneity on therapies. We then combine this with a meta-analysis of transcriptome data from distinct MSC subpopulations derived from bone marrow, adipose tissue, cruciate, tonsil, kidney, umbilical cord, fetus, and induced pluripotent stem cells derived MSCs (iMSCs). Beyond that, we investigate transcriptome differences between tissue-specific MSCs and pluripotent stem cells. Our meta-analysis of numerous MSC-related data sets revealed markers and associated biological processes characterizing the heterogeneity and the common features of MSCs from various tissues. We found that this heterogeneity is mainly related to the origin of the MSCs and infer that microenvironment and epigenetics are key drivers. The epigenomes of MSCs alter with age and this has a profound impact on their differentiation capabilities. Epigenetic modifications of MSCs are propagated during cell divisions and manifest in differentiated cells, thus contributing to diseased or healthy phenotypes of the respective tissue. An approach used to reduce heterogeneity caused by age- and tissue-related epigenetic and microenvironmental patterns is the iMSC concept: iMSCs are MSCs generated from induced pluripotent stem cells (iPSCs). During iMSC generation epigenetic and chromatin remodeling result in a gene expression pattern associated with rejuvenation thus allowing to overcome age-related shortcomings (e.g., limited differentiation and proliferation capacity). The importance of the iMSC concept is underlined by multiple clinical trials. In conclusion, we propose the use of rejuvenated iMSCs to bypass tissue- and age-related heterogeneity which are associated with native MSCs.

Keywords: heterogeneity, MSC, iMSC, iPSC, rejuvenation, epigenetics 


\section{INTRODUCTION}

Mesenchymal stem cells (MSCs) (Friedenstein et al., 1970; Caplan, 1991)/medicinal signaling cells (Caplan, 2017)/mesenchymal stromal cells (Horwitz et al., 2005) are multipotent cells with in vitro differentiation potential into mesodermal lineages such as adipocytes, chondrocytes, osteocytes, and myocytes. However, per definition in vitro cultured MSCs and their mesodermal differentiation potential are not comparable with the in vivo case (Keating, 2012; Caplan, 2017). Besides the mesodermal differentiation potential, the definition comprises plastic adherence and positivity for the surface markers cluster of differentiation (CD)73, CD90, and CD105 and negativity for CD45, CD34, CD14 or CD11b, CD79alpha or CD19, and HLA-DR surface molecules (Dominici et al., 2006).

MSCs have shown good therapeutic results in a plethora of studies and clinical trials (Keating, 2012; Jungbluth et al., 2019), yet their characteristics have not been fully clarified or defined. A central question concerns their mechanism(s) of action in therapeutic settings: Is it based on differentiation of MSCs into a target cell type or on paracrine effects triggering surrounding cells to regenerate defective tissue? Evidence exist for both (Boyle et al., 2006; Keating, 2012; Caplan, 2017; Spitzhorn et al., 2018; Jungbluth et al., 2019). Paracrine signaling has been further explored to identify the signaling molecules and exploit their therapeutic effects (Caplan and Correa, 2011; Johnson et al., 2012; Kusuma et al., 2017; Samper Agrelo et al., 2020). The heterogeneity of MSCs and the microenvironment can influence a complex interplay of paracrine and potentially autocrine effects to exert beneficial or detrimental effects, e.g., co-operation of paracrine IL1-signaling and autocrine PGE2 in MSCs and carcinoma cells inducing expression of cytokines, followed by $\beta$-catenin signaling and finally formation of cancer stem cells (Li et al., 2012). Like a double-edged sword, MSCs can promote and inhibit cancer (Lee and Hong, 2017; Galland and Stamenkovic, 2020) by various mechanisms depending on their heterogeneity and the exact dosage and timing of the treatment which have to be elucidated further to ensure secure therapies. A better understanding of MSC properties such as immunomodulation, homing to the site of injury and paracrine signaling will give rise to many therapeutic applications, e.g., bi-specific antibody therapy taking advantage of tailored MSCs constantly producing bi-specific antibodies and redirecting $\mathrm{T}$ cells to target leukemic cells (Aliperta et al., 2015; AlmeidaPorada et al., 2020). Recent publications from the Weinberg lab about epigenetic changes caused by epithelial-mesenchymal transition (EMT) distinguishing cancer stem cells (CSCs) from the non-CSC-tumor cells (Shibue and Weinberg, 2017) and by Carter and Zhao (2020) emphasize the role of epigenetics in cell fate decisions which lead to cellular heterogeneity manifested in distinct lineages and distinct differentiation and disease states.

MSCs are further characterized by predominantly beneficial immunomodulatory properties which is not in the focus of this review but described in more detail, e.g., by Chen et al. (2011); Keating (2012), Wang et al. (2016), and
Galland and Stamenkovic (2020) and with the focus on immunotherapy by Almeida-Porada et al. (2020).

MSCs from distinct sources such as bone marrow or adipose tissue differ in certain aspects, e.g., Wagner et al. (2005) found differences regarding mesodermal development and proliferation thus confirming reports of higher proliferative potential of adipose-derived MSCs by Lee et al. (2004). There were several additional studies on heterogeneity in MSCs such as one by Roson-Burgo et al. (2016) and single-cell-sequencing studies (McLeod and Mauck, 2017; Barrett et al., 2019). The singlecell-sequencing technique provides means to assess and possibly tackle intra-population heterogeneity (McLeod and Mauck, 2017; Wolmarans et al., 2021) which can vary in cell culture over time and pose problems to clinical efficacy (Phinney, 2012; Costa et al., 2021; Zha et al., 2021). Heterogeneity is also reflected in a perspective paper by authors from the FDA who regard hematopoietic reconstitution therapies with bonemarrow-derived stem cells as established but see lacking evidence for the clinical efficacy of many other stem-cell based therapies such as adipose-tissue-derived MSC treatments (Marks et al., 2017) thus hinting at a reason for the large discrepancy between the numbers of clinical trials and approval by the FDA. The phenomenon of heterogeneity investigated in all of these studies is related to the global MSC definition providing advantages and disadvantages. Keating discusses the trade-off between a simplistic global definition and potential definitions for MSC subsets (Keating, 2012).

Heterogeneity reflects predisposition for dedicated lineages as proposed by Muraglia et al. (2000) in a hierarchical model for bone marrow MSCs losing lineage potential from osteo-chondroadipogenic via osteo-chondrogenic to osteogenic precursors and excluding osteo-adipo- and chondro-adipogenic lineages. Later, reciprocality of adipogenesis and osteogenesis were reported (Chen Q. et al., 2016). Cell fate commitment of MSCs is transcriptionally regulated by various pathways and this source-associated MSC predisposition may be directed by distinct epigenetic programs (Sui et al., 2020). The nature of MSCs was further elucidated by reports on their perivascular origin in multiple organs (Caplan, 2008; Crisan et al., 2008). Furthermore, the MSC donor age plays an important role in regeneration capabilities which are better in MSCs from young donors. This is a strong argument for MSCs derived from pluripotent stem cells (iMSCs) (Barberi et al., 2005) which meanwhile have been characterized in several studies including (Diederichs and Tuan, 2014; Frobel et al., 2014) and which we found to possess a young or rejuvenated phenotype (Jungbluth et al., 2019; Spitzhorn et al., 2019). Reasons for this age and tissue dependent differences lie in distinct epigenetic programs orchestrating changes in gene expression (Frobel et al., 2014). Carter and Zhao (2020) suggest the use of novel singlecell methods for assaying epigenetic heterogeneity to elucidate processes such as differential priming for cell fate decisions among populations of stem cells.

In this study we investigate the heterogeneity of MSCs from distinct tissues and individuals reflecting distinct genetic backgrounds and ages. We further compare MSCs to MSC populations of distinct tissue origins such as urine-derived renal 
progenitor cells (UdRPCs; Rahman et al., 2020) and hepatic stellate cells (HSCs; Kordes et al., 2014). Moreover, we explore if the rejuvenation concept manifested in iMSCs can indeed reduce heterogeneity seen in native MSCs.

\section{MATERIALS AND METHODS}

\section{Sample Collection for the Meta-Analysis}

Samples for the meta-analysis were obtained from NCBI GEO (National center for biotechnology information, Gene expression omnibus) on two levels: first MSC gene expression microarray data from the same platform (Illumina Human HT-12) was downloaded to enable full comparison of expression signals. These datasets are shown in Table $\mathbf{1}$ and contain the GEO accessions GSE97311 with bone-marrow-derived aged and fetal MSCs and iMSCs (Spitzhorn et al., 2019), GSE149171 with adipose-tissue-derived MSCs (Jung et al., 2020), GSE77227 with kidney-derived perivascular stromal cells (Leuning et al., 2017), GSE77272 with tonsil-, bone- marrow-, and adipose-tissuederived MSCs (Park et al., 2016), GSE59662 with anteriorcruciate-derived MSCs (Lee et al., 2015), and GSE52841 with Wharton's jelly-derived MSCs (Sukarieh et al., 2014). Second, datasets of multiple technical platforms were downloaded for binary assessment if genes were expressed thus enabling comparison of symbols related to expressed genes. These datasets are shown in Table 2 and contain the GEO accessions GSE128281 with urine-derived renal progenitor cells (UdRPCs; Rahman et al., 2020), GSE100448 with amniotic-fluid-derived MSCs (Spitzhorn et al., 2017), and GSE152250 with hepatic stellate cells (Zhang et al., 2020).

\section{Meta-Analysis of Datasets on the Illumina Gene Expression Platform}

Datasets in form of non-normalized data listed in Table $\mathbf{1}$ were downloaded from NCBI GEO and imported into the $\mathrm{R} /$ Bioconductor (Gentleman et al., 2004) using the package lumi ( $\mathrm{Du}$ et al., 2008). Detection- $p$-values were available for all datasets except for the dataset with accession no. GSE149171 with adipose-tissue-derived MSCs (Jung et al., 2020). Detection- $p$-values for this dataset were generated by fitting a loess model to detection- $p$-values and logarithmic (base 2) expression values of the comparable dataset with accession no. GSE52841 and applying this model to predict the detection $p$-value from the logarithmic expression values of dataset GSE149171. A threshold of det-p-value $<0.05$ was employed to judge if a gene was expressed. Imported Illumina data was transformed to the $\log 2$ scale and normalized via quantile normalization. Hierarchical clustering analysis for the generation of the dendrogram was performed with the method hclust using Pearson correlation as similarity measure and complete linkage as agglomeration method and the package dendextend (Galili, 2015) for the color bars indicating the cell types. Heatmaps were generated employing the method heatmap.2 from the $\mathrm{R}$ package gplots (Warnes et al., 2015).

\section{Extension of the Meta-Analysis by Datasets on Diverse Transcriptome Platforms}

Datasets in form of Affymetrix raw data (CEL files) and summarized Illumina next-generation sequencing (NGS) data listed in Table 2 were downloaded from NCBI GEO and imported into the R/Bioconductor (Gentleman et al., 2004) using the package affy (Gautier et al., 2004) for the datasets with accession nos. GSE128281 and GSE100448 on the Affymetrix Human Primeview platform. Detection- $p$-values were calculated as described in our previous publication (Graffmann et al., 2016). A detection- $p$-value $<0.05$ was employed to identify expressed genes. For the NGS dataset GSE152250 with hepatic stellate cells a threshold of FPKM $>1$ was applied to filter expressed genes. ENSEMBL gene ids were mapped to gene symbols using the annotations from ENSEMBL Biomart version 103 (Haider et al., 2009).

\section{Venn Diagrams and Gene Ontology Analysis}

Venn diagrams were employed to dissect subsets of genes expressed at detection- $p$-value $<0.05$ (microarrays) or FPKM > 1 (NGS data). Venn diagrams were generated with the R package gplots (Warnes et al., 2015). Subsets of genes expressed exclusively in distinct MSC types were further subjected to overrepresentation analysis employing the Bioconductor package GOstats (Falcon and Gentleman, 2007). The most significant GO-terms were plotted as bar charts via the R package ggplot2 (Wickham, 2009) of the negative logarithm (base 10) of the $p$-values indicating the number of involved genes and the ratio of involved genes to the total of genes in the GO-term on a color scale.

\section{RESULTS}

\section{Comparison of MSCs of Different Sources in a Meta-Analysis}

In a meta-analysis of transcriptome data, we compared MSCs of distinct sources which includes bone marrow (BM), adipose tissue (AT), umbilical cord (UC), cruciate, tonsil, kidney, and iMSCs listed in Table 1 and also Pluripotent stem cells (PSCs). With respect to pluripotency and age, we classify samples into three meta-groups PSC, young, and adult. Embryonic stem cells (ESCs) and induced pluripotent stem cells (iPSCs) fall into the meta-group PSC, UC-derived, and fetal MSCs into the metagroup young as well as iMSCs for which we had shown the rejuvenated phenotype in a previous publication (Spitzhorn et al., 2019). The remaining samples are classified as adult. Figure 1 shows that young MSCs (yellow color bar) cluster separately from adult MSCs (blue color bar). However, in a superior cluster, young and adult MSCs are joined and cluster separately from PSCs (red color bar) of embryonic or iPSC origin. This grouping of samples reflects the differences between pluripotent and multipotent cells as manifested in their transcriptome. Adult and young MSCs fall into the same group of multipotency but 
TABLE 1 | Samples on platform lllumina Human HT-12.

\begin{tabular}{|c|c|c|c|c|c|}
\hline GSE97311_samples & GSE77272_samples & GSE52841_samples & GSE59662_samples & GSE77227_samples & GSE149171_samples \\
\hline Spitzhorn et al. (2017) & Park et al. (2016) & Sukarieh et al. (2014) & Lee et al. (2015) & Leuning et al. (2017) & Jung et al. (2020) \\
\hline Pubmedid:30885246 & Pubmedid:27224250 & Pubmedid:25129543 & Pubmedid:25729860 & Pubmedid:28191776 & Pubmedid:32961 \\
\hline aged_MSC_74y & adipose_tissue_MSC_1 & UC_SGA_MSC_1 & total_knee_arthroplasty_MSC_1 & bmMSC1 & adipose_MSC_siControl_1 \\
\hline iPSC_MSC_74y_viral & adipose_tissue_MSC_2 & UC_MSC_1 & total_knee_arthroplasty_MSC_2 & bmMSC2 & adipose_MSC_siControl_2 \\
\hline iMSC_74y_viral & adipose_tissue_MSC_3 & UC_SGA_MSC_2 & anterior_cruciate_ligament_MSC_1 & bmMSC3 & adipose_MSC_siControl_3 \\
\hline aged_MSC_62y & adipose_tissue_MSC_4 & UC_SGA_MSC_3 & anterior_cruciate_ligament_MSC_2 & kPSC1 & adipose_MSC_siDGCR8_1 \\
\hline iPSC_MSC_62y_episomal & bone_marrow_MSC_1 & UC_MSC_2 & anterior_cruciate_ligament_MSC_3 & kPSC2 & adipose_MSC_siDGCR8_2 \\
\hline fetal_MSC_3 & bone_marrow_MSC_2 & UC_MSC_3 & anterior_cruciate_ligament_MSC_4 & kPSC3 & adipose_MSC_siDGCR8_3 \\
\hline iPSC_MSC_fetal_line_1_viral & bone_marrow_MSC_3 & UC_MSC_4 & & & adipose_MSC_Early_pasage_1 \\
\hline iPSC_MSC_fetal_line_1_episomal_1 & bone_marrow_MSC_4 & UC_SGA_MSC_4 & & & adipose_MSC_Early_pasage_2 \\
\hline iPSC_MSC_fetal_line_1_episomal_2 & tonsil_MSC_1 & & & & adipose_MSC_Early_pasage_3 \\
\hline iMSC_fetal_line_1_viral & tonsil_MSC_2 & & & & adipose_MSC_Late_passage_1 \\
\hline iMSC_hESC_H1 & tonsil_MSC_3 & & & & adipose_MSC_Late_passage_2 \\
\hline H1_a & tonsil_MSC_4 & & & & adipose_MSC_Late_passage_3 \\
\hline H1_b & & & & groups: & \\
\hline H9_a & & UC: umbilical_cord & & bone_marrow_MSC & \\
\hline aged_MSC_60y & & SGA: Small for Gestational Age & & kidney perivascular stromal cell & \\
\hline \multicolumn{6}{|l|}{ aged_MSC_70y } \\
\hline \multicolumn{6}{|l|}{ fetal_MSC_1 } \\
\hline \multicolumn{6}{|l|}{ fetal_MSC_2 } \\
\hline \multicolumn{6}{|l|}{ groups: } \\
\hline \multicolumn{6}{|l|}{ bone_marrow_MSC } \\
\hline \multicolumn{6}{|l|}{ fetal_MSC } \\
\hline \multicolumn{6}{|l|}{ iMSC } \\
\hline \multicolumn{6}{|l|}{ ESC } \\
\hline iPSC & & & & & \\
\hline
\end{tabular}


TABLE 2 | Samples of amniotic-fluid-derived MSCs (AF-MSCs), urine-derived progenitor cells (UdRPCs), and hepatic stellate cells (HSCs).

\begin{tabular}{|c|c|c|}
\hline GSE100448_samples & GSE128281_samples & GSE152250_samples \\
\hline Spitzhorn et al. (2017) & Rahman et al. (2020) & Zhang et al. (2020) \\
\hline Affymetrix Human Primeview & $\begin{array}{l}\text { Affymetrix Human } \\
\text { Primeview }\end{array}$ & Illumina HiSeq 4000 \\
\hline Pubmedid:29225627 & Pubmedid:31959818 & Pubmedid:33205063 \\
\hline AF_MSC_1 & UdRPC_UM54 & HSC_US-1564046 \\
\hline \multirow[t]{17}{*}{ AF_MSC_2 } & UdRPC_UM48 & HSC_US-1564053 \\
\hline & UdRPC_UF60 & HSC_US-1564057 \\
\hline & UdRPC_UM27 & HSC_US-1564058 \\
\hline & UdRPC_UF61 & HSC_US-1564061 \\
\hline & UdRPC_UM51 & HSC_US-1564063 \\
\hline & UdRPC_UF45 & HSC_US-1564067 \\
\hline & UdRPC_UF31 & HSC_US-1564072 \\
\hline & UdRPC_UF21 & HSC_US-1564073 \\
\hline & & HSC_US-1564080 \\
\hline & & HSC_US-1564088 \\
\hline & & HSC_US-1564090 \\
\hline & & HSC_US-1564091 \\
\hline & & HSC_US-1564092 \\
\hline & & HSC_US-1564098 \\
\hline & & HSC_US-1564117 \\
\hline & & HSC_US-1564127 \\
\hline & & HSC_US-1564136 \\
\hline
\end{tabular}

AF, amniotic fluid; UdRPC, urine-derived renal progenitor cell; HSC, hepatic stellate cell.

are distinguishable between each other pertaining to age-related heterogeneity within MSCs. Interestingly, kidney perivascular stromal cells cluster with adult MSCs thus confirming the common nature of MSCs and pericytes as suggested by Caplan (2008) based on the study by Crisan et al. (2008).

\section{Common and Distinct Gene Expression Signatures}

We set out to further investigate heterogeneity between distinct MSC types by comparing gene expression (detection$p$-value $<0.05$ ) on the same technical Illumina Human HT-12 microarray platform. The venn diagram in Figure 2A shows that MSCs of distinct origin share a large common gene signature of 9966 genes but differ in tissue-type specific gene signatures (Supplementary Table 1A). The two most significantly overrepresented GO terms (Biological Process) in the common 9966gene signature (Supplementary Table 1G) are primary metabolic process $(p=6 \mathrm{E}-108)$ and cellular macromolecule localization $(p=1.1 \mathrm{E}-96)$.

Subsets of the venn diagram are dissected to distinct types containing smaller numbers of genes: fetal MSCs-401, iMSCs374, adipose-tissue-MSCs-29, bone-marrow-MSCs-130, and UCMSCs-558. Over-representation analysis of Gene ontologies (GOs) in the specific subsets revealed GO terms of which the 20 most significant are listed here. These GO terms reflect the heterogeneity characterized by the specific original microenvironment of the MSCs: significant GO terms in fetal MSCs relate to developmental and morphogenetic processes
(Figure 2B and Supplementary Table 1B), significant GO terms in UC-MSCs relate to defense/inflammatory response, lung development, gastrulation (Figure 2C and Supplementary Table 1C) and significant GO terms in iMSCs relate to hormone metabolism, neuronal development, interferon, and TNF signaling (Figure 2D and Supplementary Table 1D). While these MSCs are associated with the young, developmental phenotype, Figure 3 demonstrates that the most significant GO terms in adult MSCs, in adipose-tissue and bone-marrowderived MSCs also reflect the tissue of origin. The subsets of genes exclusively expressed in adipose tissue-derived MSCs (29 genes in Figure 3A and Supplementary Table 1E) and bone-marrow-derived MSCs (130 genes in Figure $\mathbf{3 B}$ and Supplementary Table 1F) were analyzed here. Figure 3A reveals that the most significantly over-represented GO terms in adipose tissue-derived MSCs include vascular, muscle, and neuronal development which may be considered characteristic for adipose tissue. Figure $3 \mathbf{B}$ reveals that the most significantly over-represented GO terms in bone-marrow-derived MSCs include skeletal system and limb development pointing at osteogenic properties.

\section{Tissue of Origin Determines Heterogeneity of Amniotic-Fluid-Derived MSCs, Urine-Derived Renal Progenitor Cells and Hepatic Stellate Cells}

In a follow-up analysis we aimed at extending the transcriptome comparison of MSCs to MSC populations of distinct tissue origins measured on different microarray platforms and by RNAseq. Figure 4 shows this comparison with additionally amniotic-fluid-derived MSCs (AF-MSCs), urine-derived renal progenitor cells (UdRPCs), and hepatic stellate cells (HSCs). We characterized UdRPCs in a previous publication (Rahman et al., 2020), these SIX2-positive renal progenitor cells meet the criteria defined for MSCs. HSCs are activated upon injury and inflammation in the liver leading to fibrosis (Gressner and Weiskirchen, 2006; Wruck et al., 2017; Driscoll and Patel, 2019). Furthermore, an immune-modulatory beneficial effect of MSCs on HSCs has been described (Parekkadan et al., 2007; Driscoll and Patel, 2019). Beyond that, HSCs themselves have been characterized as MSCs with the ability to reduce liver fibrosis (Kordes et al., 2014). We used the genes expressed in common in MSCs on the Illumina platform (9966 genes, Figure 2A) and compared them with genes expressed in AF-MSCs, UdRPCs, and HSCs (Figure 4A). The resulting subsets of genes exclusively expressed in AF-MSCs (301 genes in Figure 4A), UdRPCs (476), and HSCs (2280) were analyzed for over-representation of GO terms (Supplementary Table 2). The most significantly overrepresented GO terms in AF-MSCs include developmental and signaling processes which may be considered characteristic for embryonic development (Figure 4B), the most significantly overrepresented GO terms in UdRPCs include lipid transport and detoxification pointing at renal properties (Figure 4C) and the most significantly over-represented GO terms in HSCs include metabolic, cristae, and mitochondrial processes pointing at liver characteristics (Figure 4D). 


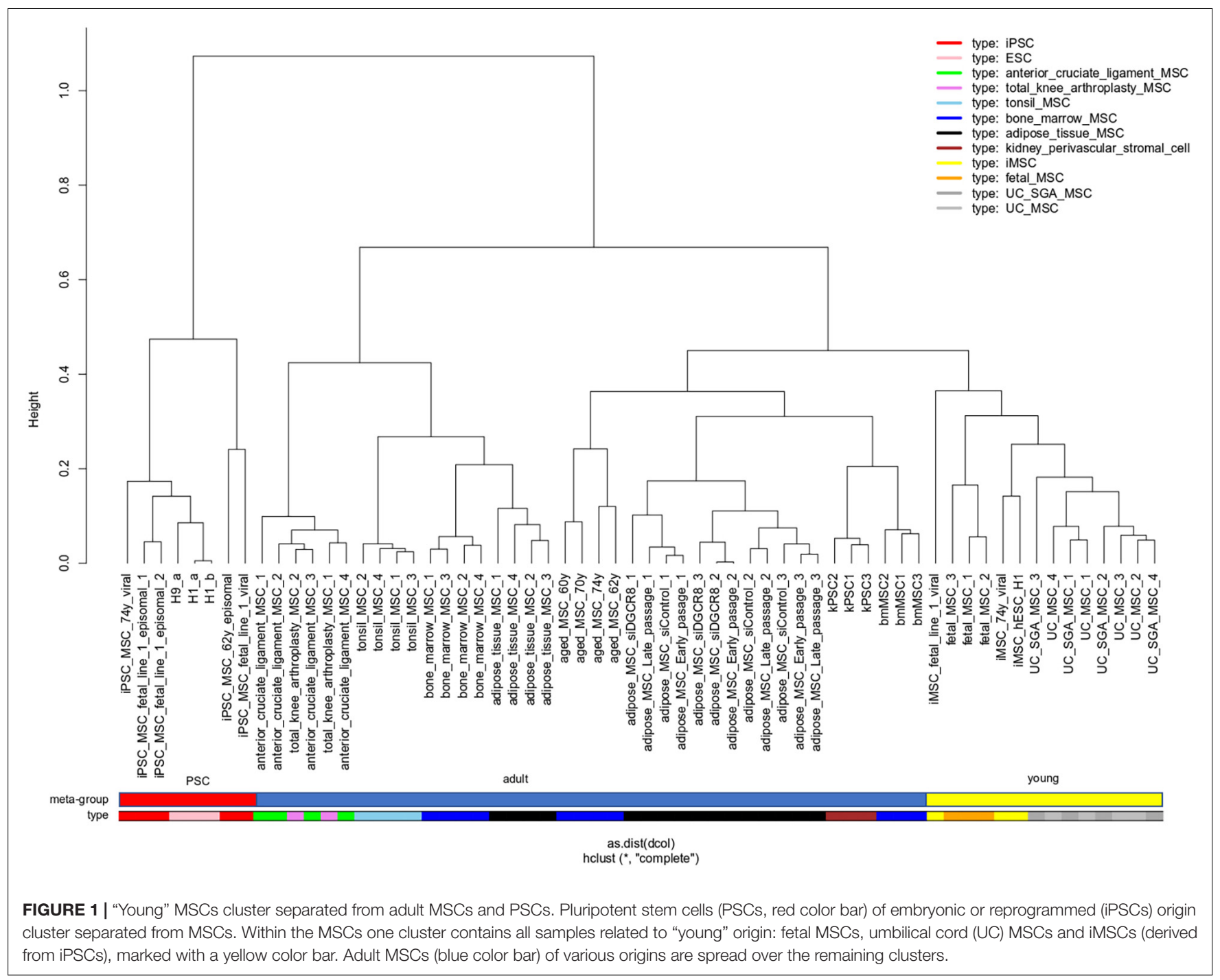

\section{Characteristics of MSCs Derived From Induced Pluripotent Stem Cells}

In our previous work we have reprogrammed fetal MSCs to iPSCs and then differentiated these back into MSCs, so called iMSCs. These iMSCs exhibited the typical MSC characteristics such as plastic adherence, spindle-shaped morphology, expression of cell surface markers CD73, CD90, and CD105 as well as PDGFR $\beta$ by parallel absence of hematopoietic and pluripotency markers such as OCT4. These iMSCs had a similar pattern of secreted molecules as native MSCs and showed a typical MSC-like differentiation capacity into adipogenic, chondrogenic, and osteogenic lineage in vitro (Spitzhorn et al., 2018, 2019). In addition, we previously showed that iMSCs are reset to a rejuvenated phenotype with a much reduced heterogeneity compared to native tissue-specific MSCs. To gain further insights and confirmation of similarities between iMSCs and native MSCs, we investigated the Biological processes present in the GO terms over-represented in the exclusive subsets of young MSC types iMSCs, fetal MSCs, and UC-MSCs from the venn diagram in
Figure 2A. Figure 5 shows that there are overlapping overrepresented GO terms of iMSCs with those of MSCs of young origin. The overlap is associated with developmental processes (Figures 5A-C). Thus, the heterogeneity between iMSCs, fetal MSCs, and UC-MSCs manifested in distinct expressed genes eventually leads to common developmental biological processes.

The process of MSC rejuvenation refers to a process in which the cells are reversed into a more embryonic state or the suppression of aging-inducing processes. Besides the generation of iMSCs, other methods have been described to induce this rejuvenation process in MSCs. MSCs can be either completely reprogrammed to iPSC or can be partially reprogrammed. These processes lead to changes in DNA methylation, histone composition, and epigenetic patterns. An alternative approach is the treatment of the cells with specific microRNAs leading to a rejuvenated stage. A further strategy for inducing rejuvenation events is management of reactive oxygen species (ROS levels). Several substances such as ascorbic acid or lactoferrin have been described to be able to reduce ROS levels and delay 

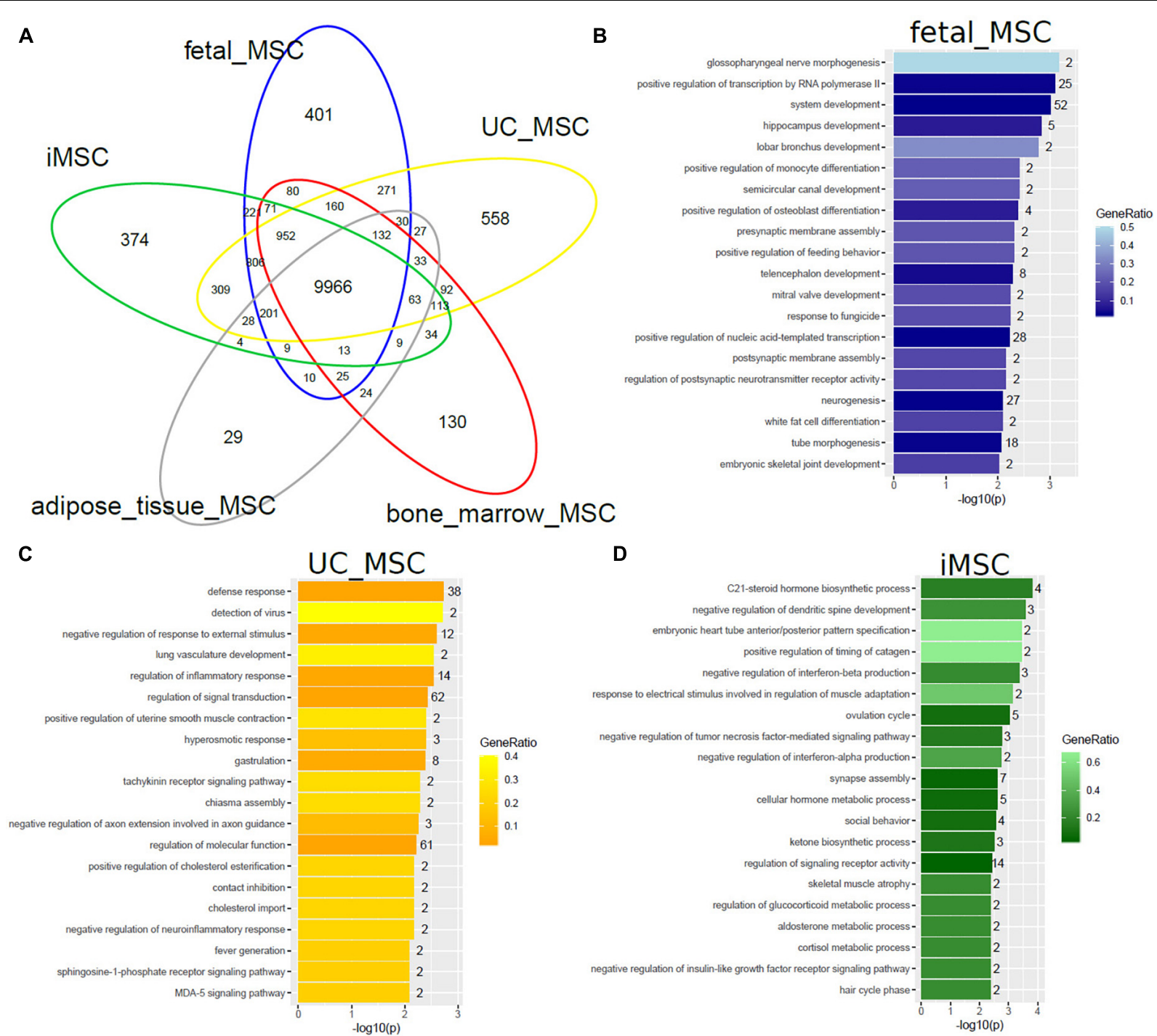

FIGURE 2 | MSCs of distinct origin share a large common gene signature but differ in smaller specific gene signatures. (A) The Venn diagram comparing gene expression in five MSC datasets on the Illumina Human HT-12 platform shows that most (9966) genes are expressed (detection- $p$-value < 0.05) in common. Specific to distinct types are subsets containing smaller numbers of genes: fetal MSCs-401, iMSCs-374, adipose-tissue-MSCs-29, bone-marrow-MSCs-130, and UC-MSCs-558. Over-representation analysis of Gene ontologies (GOs) in the specific subsets revealed GO terms of which the 20 most significant are listed here. (B) Significant GO terms in fetal MSCs relate to developmental and morphogenetic processes. (C) Significant GO terms in UC-MSCs relate to defense/inflammatory response, lung development, gastrulation. (D) Significant GO terms in iMSCs relate to hormone metabolism, neuronal development, interferon, and TNF signaling.

cellular senescence. Another relevant feature in aged cells is declining mitochondria function. Thus, several approaches target to improve mitochondria function in aged cells by upregulation of key proteins such as HSPA1L or FGF21. Also, the specific over-expression of key transcription factors such as FOXP1, YAP, or FOXD1 support the rejuvenation process. Another angle for attenuating aging is related with autophagy management. Inhibition of pathways such as mTOR can increase autophagy mechanisms and as such support protein homeostasis which is diminished in aged cells (Zhou et al., 2020). A recently described method for rejuvenation of human MSCs employs extra cellular vesicles collected from infant MSCs which are used to treat MSCs from elderly persons. In response to this treatment downregulation of ROS production in the elderly cells was observed and furthermore a better functionality regarding their ability in decreasing necrotic areas in diabetic mouse models was observed (Khanh et al., 2020).

\section{Cluster Analysis With Aging-Rejuvenation- and Epigenetics-Related Gene Signatures}

We further aimed at identifying factors contributing toward the heterogeneity phenotype typical of MSCs and their reversion to the rejuvenated state associated with iMSCs. Figure 6A shows cluster analysis and heatmap using a gene signature 

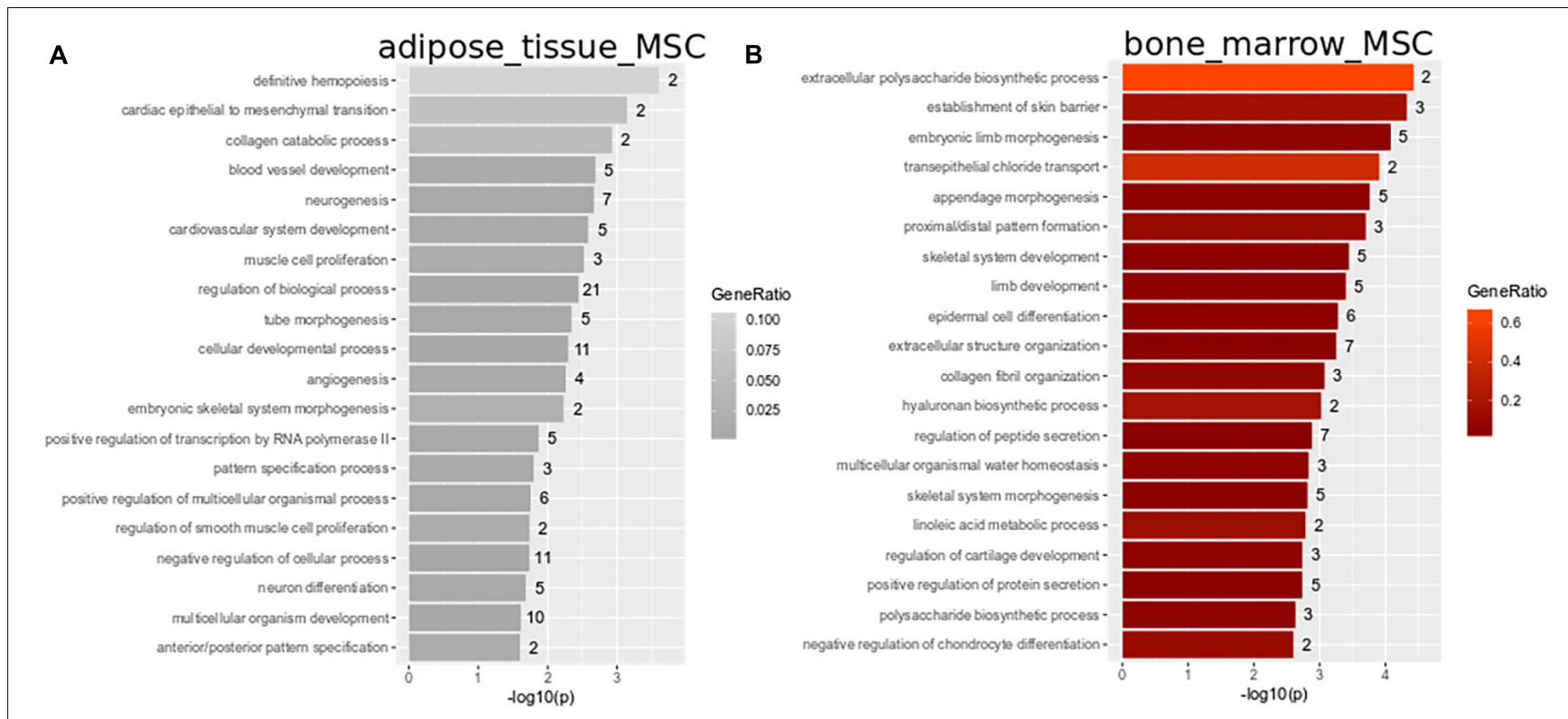

FIGURE 3 | Heterogeneity in adipose-tissue and bone-marrow-derived MSCs reflects tissue of origin. Subsets of genes exclusively expressed in adipose tissue-derived MSCs (29 genes in Figure 2A) and bone-marrow-derived MSCs (130 genes in A) were analyzed for over-representation of GO terms. (A) Most significantly over-represented GO terms in adipose tissue-derived MSCs include vascular, muscle and neuronal development which may be considered characteristic for adipose tissue. (B) Most significantly over-represented GO terms in bone-marrow-derived MSCs include skeletal system and limb development pointing at osteogenic properties.

which we found to be associated with aging and rejuvenation of MSCs at the transcriptome level in our previous publication (Spitzhorn et al., 2019). The analysis resulted in distinct clusters of PSCs and iMSCs with fetal and UC-MSCs. In Figure 6B we retrieved an epigenetics-related gene signature from the publication by Avgustinova and Benitah (2016) and could demonstrate distinct clusters of PSCs and iMSCs with fetal and UC-MSCs which, however, were a bit more fragmented than in Figure 6A.

\section{Role of Epigenetics in Heterogeneity of MSCs}

Epigenetic modifications are crucial for determining the gene expression profile of a given cell type. They are defined as mechanisms which enable or prevent access of the transcription machinery to genomic loci in a heritable and at the same time flexible manner (Bernstein et al., 2007). The most commonly analyzed factors in this regard are DNA methylation (DNAme) which occurs at chromosomal regions where CpG dinucleotides are overrepresented (Gardiner-Garden and Frommer, 1987) and histone modifications (Strahl and Allis, 2000). Promoter associated DNAme suppresses gene expression while histone modifications act as activating or repressing factors depending on the context and the nature of the modification (Bernstein et al., 2007).

Epigenetic factors have been described to regulate MSC heterogeneity in two distinct ways: (1) tissue- specific gene expression patterns and (2) age-specific gene expression patterns (Avgustinova and Benitah, 2016; Sui et al., 2020). Together, these two layers define the differentiation and regeneration potential of MSCs.

Most analyses of the epigenetic landscape of MSCs have been carried out by comparing BM and AT MSCs, which have distinct differentiation potentials (Schellenberg et al., 2011; Xu et al., 2017). While BM MSCs readily differentiate into the osteogenic lineage, this potential is reduced in AT MSCs, which favor the adipogenic differentiation. DNAme analysis of the promoters of key transcription factors such as runt-related transcription factor 2 (RUNX2) and peroxisome proliferator activated receptory $(P P A R \gamma)$ revealed an opposing profile with RUNX2 being demethylated in BM MSCs and methylated in AT MSCs while $P P A R \gamma$ was unmethylated in these cells and methylated in BM MSCs (Xu et al., 2017). Similar results were obtained when including MSCs derived from other tissues (Reinisch et al., 2015). Interestingly, only BM derived MSCs differentiate efficiently into chondrocytes and are capable of building hematopoietic niches after transplantation which has been related to their special gene expression and epigenetic profile (Reinisch et al., 2015).

Gene ontology analysis of the differentially methylated regions in BM vs. AT MSCs revealed that they correlate with the distinct functions of the cells similarly to the results we obtained when comparing the gene expression profiles of various MSCs (Figure 1; Schellenberg et al., 2011). Interestingly, the differential methylation of only 2 specific $\mathrm{CpG}$ sites reliably distinguishes between MSCs derived from BM and from AT (de Almeida et al., 2016).

The equilibrium between opposed differentiation pathways and self-renewal is not only mediated by DNAme but also by histone modifications. It has been shown that high levels of the 


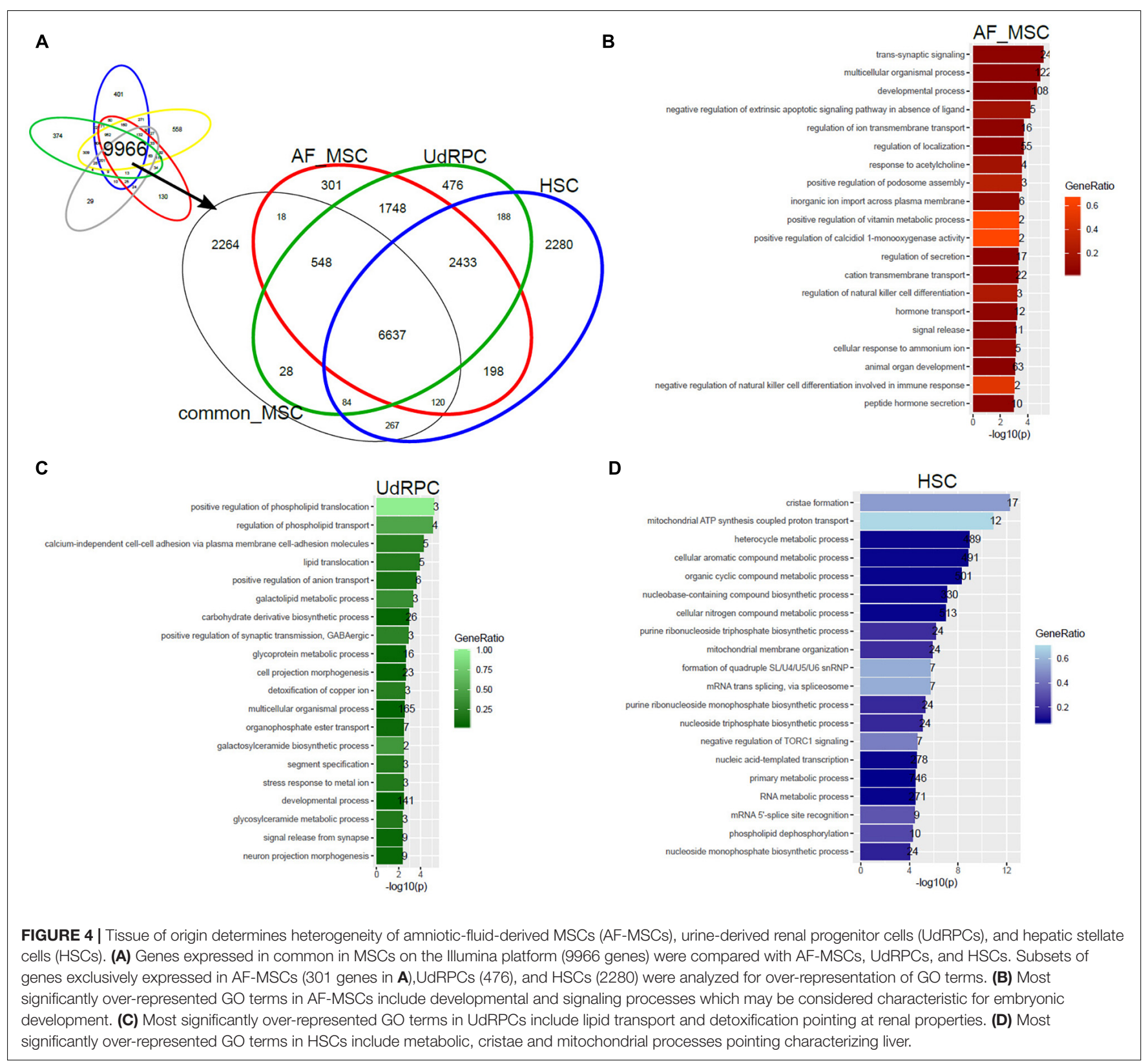

Polycomb complex 2 factor Enhancer of Zeste 2 (EZH2) favor adipogenesis of BM MSCs (Hemming et al., 2014; Jing et al., 2016), while its antagonist lysine demethylase 6A (Kdm6a), as well as SET-domain-containing 2 (Setd2) mediated H3K36me3 and Ashl1 mediated $\mathrm{H} 3 \mathrm{~K} 4 \mathrm{me} 3$ are necessary to maintain the osteogenic differentiation potential (Wang et al., 2018; Yin et al., 2019).

When we analyzed the expression level of a panel of epigenetic factors described by Avgustinova and Benitah (2016) in our data set, we saw a good clustering according to tissue type as well as age (Figure 6B).

With aging, the differentiation potential of BM MSCs changes from osteogenic to more adipogenic which is associated with impaired bone regeneration and a higher risk for osteoporosis
(Chen Y.-H. et al., 2016; Ganguly et al., 2017). This is related to an increase in EZH2 and H3K27me3 levels (Chen Y.-H. et al., 2016). In addition, the DNAme profile of MSCs changes profoundly during aging. In BM MSCs regions with repressive chromatin marks tend to gain DNAme comparable to observations made in blood cells, while open regions marked by H3K4me1 loose DNAme during aging (Fernández et al., 2015).

To overcome age-induced impairments of MSCs, we and others have derived MSCs from iPSCs, so called iMSCs which have a rejuvenated transcriptome and epigenetic profile (Figures 6A,B; Frobel et al., 2014; Spitzhorn et al., 2019) and thus promise to be particularly suitable for regenerative therapies.

Regarding the therapeutical application of MSCs, a third layer of heterogeneity is added by cell culture induced replicative 

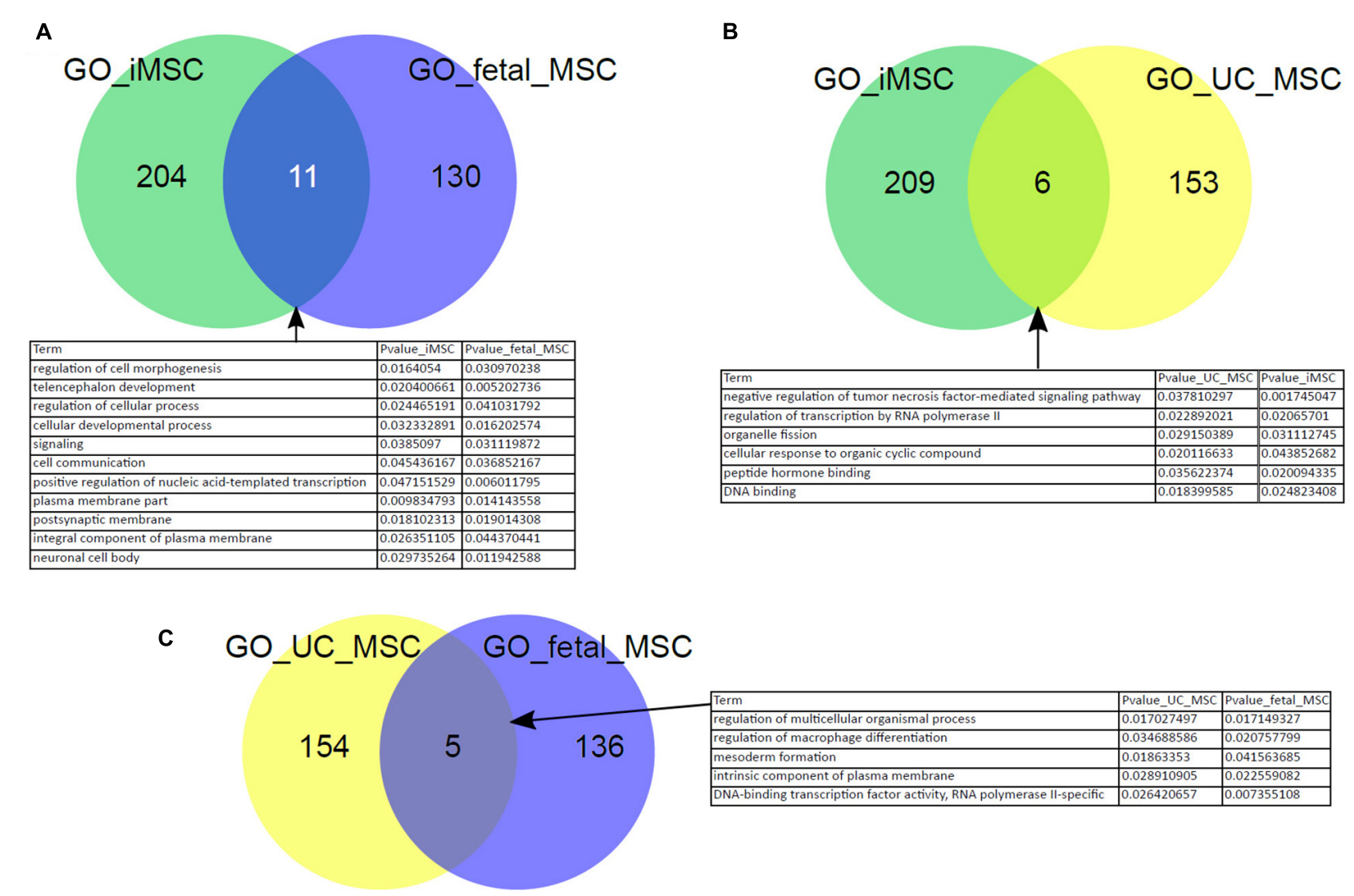

FIGURE 5 | Characteristics of iMSCs overlap with those of MSCs from young origin. Overlapping over-represented GO terms between iMSCs, fetal MSCs, and UC-MSCs were identified. The overlap is connected to developmental processes (A-C).

senescence. MSCs do not all have the same proliferation and differentiation capacity in vitro and over time the highly proliferative fibroblastoid colony forming units decline continuously from initially $20 \%$ to $1 \%$ after 2 months of cultivation (Schellenberg et al., 2011). Based on their DNA methylome, cells can be clearly separated according to their tissue of origin even after several weeks of culture. However, focusing only on senescence-associated genes, the tissue differences recede behind the senescence associate differences (Schellenberg et al., 2011). This is not due to chromosomal aberrations but to DNAme changes which were significantly enriched in regions with repressive histone modifications, namely $\mathrm{H} 3 \mathrm{~K} 9 \mathrm{me} 3, \mathrm{H} 3 \mathrm{~K} 27 \mathrm{me} 3$ as well as EZH2/Polycomb target regions (Schellenberg et al., 2011), which is similar to the observations made during aging of MSCs.

MSC function is crucial for tissue homeostasis and in this regard their heterogeneity presents advantages as well as disadvantages. On the one hand, a defined tissue related transcription profile points to highly adapted cells that are perfectly prepared for the requirements of the given tissue. On the other hand, aging-related heterogeneity shows that the transcriptional control gets impaired over time. This does not only reduce the capability of MSCs to maintain homeostasis but also is a risk factor for disease development as the propagation of a defective transcriptional program during cell division of MSCs increases the risk of developing malignancies (Sui et al., 2020).

\section{Impact of MSC Heterogeneity in Pre-clinical and Clinical Trials Application of MSCs in Clinical Trials}

Initially, MSCs were described to be supportive in the generation of new blood cells (hematopoiesis) (Maximow, 1924). Because of their immunomodulatory capacity, MSCs are widely used in the treatment of graft versus Host disease (GvHD; Le Blanc et al., 2008). They have a multilineage differentiation potential as manifested in their ability to evolve into cells from the mesodermal lineage such as bone, cartilage, and fat (Dominici et al., 2006; Crisan et al., 2008; Fitzsimmons et al., 2018). Furthermore, various reports attest that MSCs are also able to form other cell types in vitro or in vivo, such as pancreatic $\beta$ cells, cardiomyocytes, or liver cells (Segers and Lee, 2008; Cho et al., 2018; Spitzhorn et al., 2018). MSCs create a special microenvironment by secreting chemokines, growth factors, extracellular vesicles, and cytokines with immunomodulatory effect and additionally residential tissue specific progenitor cells are guided to the area of regeneration/injury (Galipeau and Sensébé, 2018; 

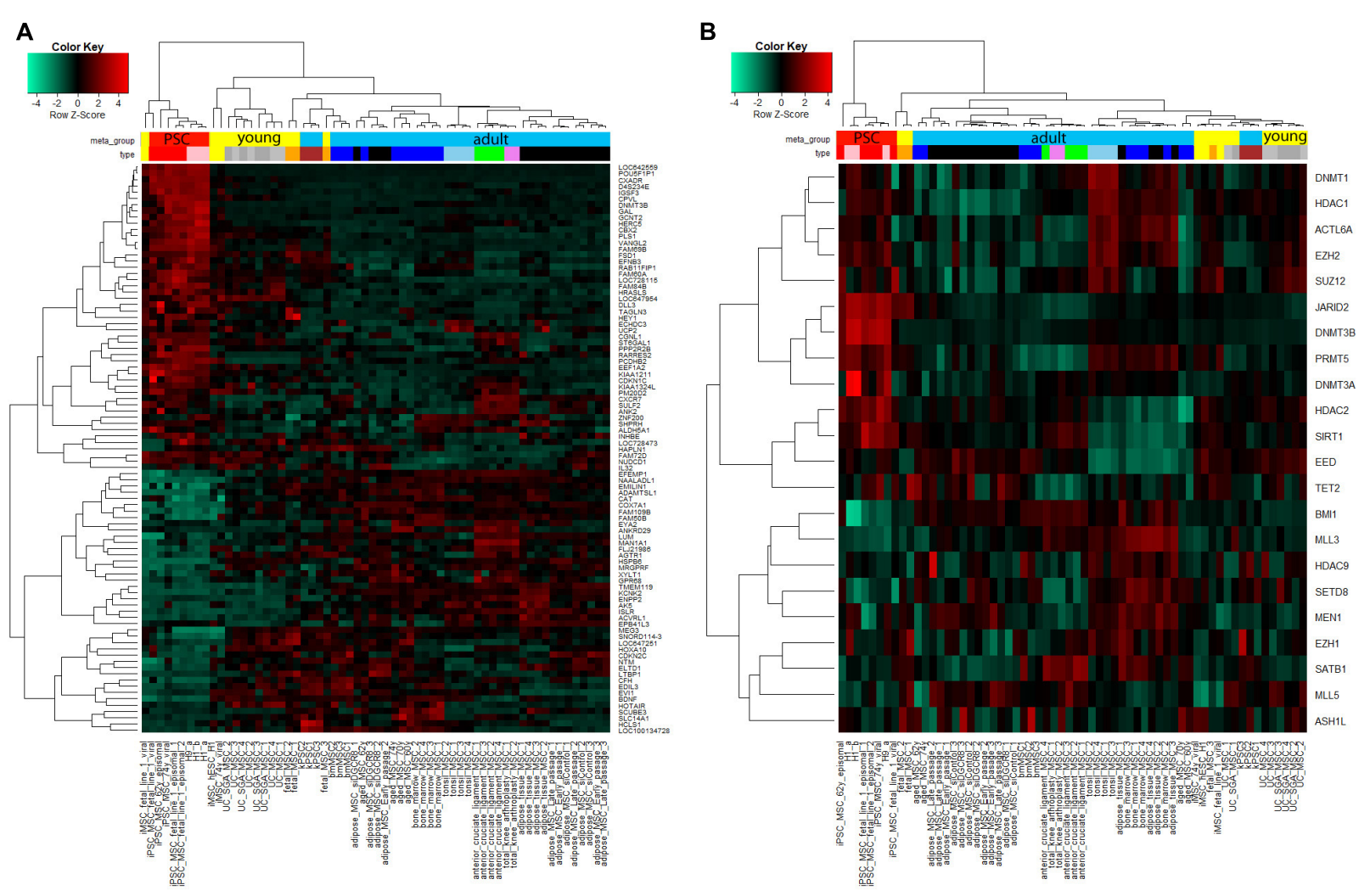

FIGURE 6 | Cluster analysis displays agglomeration of young (iMSCs, fetal, and UC-MSCs) MSCs in epigenetics-related gene signatures. (A) Heatmap using the aged-rejuvenation gene signature from our previous publication (Spitzhorn et al., 2017) yields coherent clusters of PSCs and iMSCs with fetal and UC-MSCs.

(B) Also the heatmap using the epigenetics-related gene signature from the publication by Avgustinova and Benitah (2016) yields coherent clusters of PSCs and iMSCs with fetal and UC-MSCs, however, a bit more fragmented than in (A).

Harrell et al., 2019; Rodríguez-Fuentes et al., 2021). Their secretory profile allows MSCs to support regeneration activities by the release of for example HGF, VEGF-A, PDGA, and a plethora of interleukins (Fitzsimmons et al., 2018). By sensing and reacting to environmental factors MSCs can influence the immune system by supporting T-helper 2 cells response. In addition, MSCs can influence the process of antigen-presentation and can decrease expansion and recognition of T-cells (Caplan, 2017). A big advantage in the use of MSCs for cellular therapies are their low expression levels of human antigen class II Molecules, thus allogenic transplantation of cells without HLA matching is possible (Bloor et al., 2020).

All these characteristics make MSCs very promising for the use in innovative therapeutic approaches. In line of this, MSCs are or have been used in over 1,000 clinical trials for versatile applications in the various organs and diseases. ${ }^{1}$ Neurological and orthopedic (joint) impairments, cardiovascular disease, and GvHD are the top four indications for MSC applications (Kabat et al., 2020). China and United States are the main drivers of MSC-based trials (Rodríguez-Fuentes et al., 2021). By the year 2020 there have been more than 300 completed clinical trials using MSCs (Levy et al., 2020). Reacting to the current pandemic

${ }^{1}$ https://clinicaltrials.gov situation, first studies using MSCs in the treatment of COVID-19 have been started and show their broad potential use cases (Leng et al., 2020; Shetty, 2020).

In total, MSC therapies have been shown to have an acceptable safety profile with parallel beneficial therapeutic effects in several clinical studies. The number of clinics offering stem cells therapy is constantly increasing; so is the number of companies working on MSC related products. But his also comes along with malpractice and misuse of those cells (Zhou et al., 2021). Although many clinical trials have shown the safety of transplanted MSCs, the beneficial outcome is often not clearly shown. In contrast to the immense research undertaken in MSC-based clinical trials, only a small number of MSC-based products or therapies have been approved to date. MSCs were successfully used in pioneer studies for cellular therapies in the human system in 1995, where they were autologously transplanted to support the hematological recovery (Lazarus et al., 1995). Their immunosuppressive activity is frequently exploited in transplantation settings to diminish the risk of organ rejection or graft-versus-host-disease (GvHD; Fitzsimmons et al., 2018). A phase 3 clinical trial where MSCs are used for treatment of complex perianal fistulas in Crohn's Disease (Panés et al., 2016) is one of the most successful latest trials (NCT01541579). Although the mode of action is not fully proven but indicated to be related to immunomodulatory capacity, this 
MSCs based product, Alofisel, was approved by the European Medicines Agency in 2018 (Levy et al., 2020). Over the course of time ten MSC products have been approved (numbers of products in brackets) for use in fistulas in Crohn's disease (2), GvHD (2), subcutaneous tissue defects (1), amyotrophic lateral sclerosis (1), knee cartilage defects (1), spinal cord injury (1), critical limb ischemia (1), and acute myocardial infarction (1). Thereof one product is approved in Europe, one in Canada and New Zealand, one in Japan, five in South Korea, and one in India (Levy et al., 2020). We refer to Table $\mathbf{1}$ from the publication of Levy et al. (2020) for a summary of the application, administration routes and clinical efficacy of MSCs in clinical trials for different diseases. Levy et al. (2020) - referring to Galipeau and Sensébé (2018) - report a typical dose of 1-2 million MSCs/kg for intravenous injection into humans.

Most MSC-based clinical trials use adult autologous or allogeneic MSCs from the iliac crest (bone marrow), placenta, or adipose tissue (Rodríguez-Fuentes et al., 2021). Allogeneic MSC transplantation is evaluated to be the most promising route due to larger possible scales and use of less invasive procedures. With compliance to GMP standards this possibility is a safe, accessible treatment option for the patients (Rodríguez-Fuentes et al., 2021) although native MSCs are rare and only accessible by complex processes in vivo (MacQueen et al., 2013). The usual route of MSC administration is intravenous transfusion followed by local injection (Rodríguez-Fuentes et al., 2021). The most effectives doses were calculated to be around $150 \times 10^{6}$ cells per patient (Kabat et al., 2020), in line with the 1-2 million cells/kg suggested by Galipeau and Sensébé (2018).

In addition to the before mentioned use cases, other abilities of MSCs are under investigation. Since MSCs can evade the host immune system partially, MSCs have been employed as drug delivery vehicles for example in anti-cancer treatment since they are also able to home into tumor tissue (Sasportas et al., 2009). Many of these MSC-based cancer treatments have reached the clinical stage now (Levy et al., 2020). In contrast to other cellular therapeutic agents, MSCs strategies do not only rely on cell contact or differentiation effects but also on their paracrine effector function (Levy et al., 2020). Since a large number of studies is only relying on the released molecules, the cells direct regenerative and differentiation potential is not necessarily in the focus of such investigations (Wilson et al., 2019). This is why the concept of "medicinal signaling cells" has been introduced (Caplan, 2017). Exploiting MSC secreted factors or extracellular vesicles is of high potential but the engineering and scaling process of such released factors have to be established in a standardized manner to enable broad clinical use (Phinney and Pittenger, 2017).

\section{MSC Heterogeneity Is a Multi-Level Issue Which Influences the Outcome of Clinical Trials}

The general problem of clinical trials is the long certification process which leads to the fact that the final end-therapeutic agent is $5-10$ years behind the latest research advances. Although over 1,000 clinical trials using MSCs have been performed, many MSC therapies fail in the later clinical stages, which is reflected by the fact that so far only ten MSC-based products have reached (partial) market approval. One of the main reasons for this are the many layers of heterogeneity associated with the use of MSCs: inter-cellular differences and various cell (sub)populations, inter-tissue differences and inter-donor or inter-recipient variations. Another layer of heterogeneity is introduced by the researchers themselves by the use of different protocols for cryopreservation, up-scaling and administration (Tanavde et al., 2015; Levy et al., 2020).

\section{Cells/Tissues}

A big hurdle which needs to be overcome to finally have a broad success in MSC-based therapy is the cells' intrinsic heterogeneity. It is well described that MSCs are a heterogeneous cell population (O'Connor, 2019) and is a big challenge for researchers to develop robust therapy strategies (Zha et al., 2021). Cellular heterogeneity has impacted the effectiveness of MSC therapies in animal models and has been cited as a possible factor contributing to the variability in treatment outcomes of MSC therapies in clinical trials (O'Connor, 2019). First of all the tissue and the donor (age, health status) dictate MSC quality. The difference in tissue-specific MSCs may result from the present varying microenvironments (niches). MSCs preparation from distinct tissues have shown a certain degree of heterogeneity as revealed in the meta-analysis included in this study and by whole transcriptome data and single cell RNA sequencing (Huang et al., 2019; Liu et al., 2019; Sun et al., 2020). Clinical efficacy is also influenced by the fact that MSCs from distinct tissue sources also exhibit variation in their differentiation lineage potential (Zhou et al., 2021). Not only inter-tissue differences have been shown but even amongst sampling sites of the same tissue, the same niche has led to different outcomes (Costa et al., 2021). One key finding in MSCbased therapeutic use it that there is a transfer gap between the surface marker expression which is associated with in vitro function and the corresponding in vivo effect (Wilson et al., 2019). Depending on the tissue MSCs are derived from they exhibit varying immunophenotype and differentiation potential. An example for this is the finding that MSCs from the dental pulp predominantly differentiate into neuronal cells (Suchanek et al., 2009) whereas MSCs from birth-associated tissues such as amnion and umbilical cord have a high prevalence to evolve in hematopoietic direction (Sivasubramaniyan et al., 2012). Bone marrow derived MSCs have a great differentiation capacity into osteogenic and chondrogenic direction (Vinardell et al., 2012), whereas MSCs from adipose tissue are more prone for adipogenic differentiation (Lotfy et al., 2014). This is why, in addition to the classical MSCs markers other MSC subpopulations are under investigations CD271+, CD49f+, CD146+, and Stro-1+ (Zha et al., 2021). Addressing molecular heterogeneity amongst various MSC preparations is necessary to ensure a high degree in efficiency and safety of MSC therapies and will support assessment of proper cell quality control, scaling strategies, and application therapeutical strategies (O'Connor, 2019).

\section{Variabilities Between Donors}

In addition to this inter-cellular multiplicity, it has been shown that potency and the quality of MSCs has huge inter-donor 
variation (Xin et al., 2010). The function of MSCs is influenced by donor parameters such as age, health status, sex, and the region the MSCs are derived from Zha et al. (2021). From the donor side, MSC quality is hampered for example in disease conditions such as diabetes, rheumatoid arthritis, or Parkinson's disease (Costa et al., 2021). For example it was shown that MSCs derived from the bone marrow of patients suffering from osteoarthritis had a lower multi-lineage differentiation potential (especially into cartilage direction) and an altered cell surface marker pattern than from healthy donors (Čamernik et al., 2020). Precisely in the immunosuppressive actions of MSCs, significant inter-donor variation have been reported (Ketterl et al., 2015). Although MSCs have been used in many clinical trials for immune-related diseases with promising results, the individual immune reaction can be very heterogeneous (Carvalho et al., 2019). These differences in the host immune reaction in relation to cellular therapies are an important factor (Levy et al., 2020). Many studies have underlined the variable proliferation and differentiation capacity as well as their secretory profile and their anti-tumor effect when emanating from distinct tissues or donors (Costa et al., 2021). The typical clinical scenario is dominated by elderly patients. For these people, the potential of their MSCs is diminished because of cellular aging-associated effects such as senescence, genome instability, oxidative stress, and DNA damage (Zhou et al., 2008; Reuter et al., 2010; Moskalev et al., 2013; Yang et al., 2018). All these aging-related characteristics negatively influence the differentiation capacity and functionality and thereby the ultimate therapeutic performance (Oreffo et al., 1998; Stolzing and Scutt, 2006; Wagner et al., 2009).

\section{Handling of MSCs in Culture}

The described levels of MSC heterogeneity are multiplied by differences in the various researcher- triggered parameters in the in vitro stages prior to admission (Madsen et al., 2017). MSCs derived from primary tissue sources are restricted in their use since long term culture is influencing their proliferation and differential potential as well as their phenotype (Wagner et al., 2008). Clinical trials require large numbers of MSCs, this results in the need of prolonged in vitro expansion which results in early senescence and altered gene expression patterns which have a negative effect on the therapeutic potential (Li et al., 2020). The cell isolation processes, culture conditions such as medium, substrates, and $\mathrm{O}_{2}$ concentration, have significant influence on the characteristics of MSCs as well as cryopreservation and thawing routines are critical steps influencing quality. The culture conditions have to mimic the specific stem cell environment. MSCs can be cultured as clones, however, heterogeneity was observed to be inter- and intra-clonal (Costa et al., 2021; Zhou et al., 2021). Despite the cellular variations, another very important factor is the handling of the MSCs including, expansion, freezing, thawing, and administration processes since all these steps can have significant influence on the therapeutic outcome (Levy et al., 2020). Handling factors such as injection volume, needle, injection site, buffer used, all have different physical properties such as shear stress and all contribute toward heterogeneity (O'Cearbhaill et al., 2014). All of these MSC culture condition and parameters have been shown to influence global cellular signatures and therefore need to be critically investigated for optimal design of the scaling process for subsequent clinical success (O’Connor, 2019).

\section{Strategies to Overcome These Challenges}

At first the in vivo study management in pre-clinical studies has to be improved to enable more successful clinical trials. Especially in the orthopedic field mostly small animal models are used and therefore the human scenario is insufficiently reflected. The big gap from pre-clinical to clinical stage is partially caused by the fact that (small) animal models require a much lower cell number as compared to the human system where prolonged in vitro culture of MSCs are needed (Wilson et al., 2019).

Although there are many promising results in MSC-based trials, other trials were discontinued after disapproval by the FDA due to poor quality of the controls, and heterogeneity of MSCs with regard to stability, differentiation, and migratory potential (Conrad et al., 2009; Haga et al., 2015). Other drawbacks in the use of primary MSCs is the logistical and financial complex process of continual donor identification, qualification, and recruitment. Furthermore, large cell quantities are needed for cellular therapy which makes it necessary to culture the initially obtained cells in vitro which can have also negative impacts such as reduced stemness (Bloor et al., 2020). Clinical results have already indicated that MSCs which have undergone minimal expansion prior to transplantation perform better in the case of GvHD (von Bahr et al., 2012). In contrast to normal biological and chemical-based drugs, MSC-based therapies are dynamic and more complex (Levy et al., 2020). Biological drugs require a clear identification, purity and the efficacy of the product. The active substance in the cellular product is clear to name. Although strategies are there to increase cell purity, authorities so far do not necessarily insist to have a completely pure product. There seems to be a general acceptance of heterogeneity within a cellular product which makes the whole landscape of MSC-based clinical trials very complex. Nevertheless, the most important aspect is to define the exact mode of action of the cellular agent including the upstream and downstream molecular events (Wilson et al., 2019). Furthermore, MSC subpopulation identification and understanding as well as mastering the robust standardized manufacturing process of MSCs are important aspects for the establishment of more MSC-based therapies (Levy et al., 2020; Zha et al., 2021). Many MSC based clinical trials are company driven. This is associated with lack of fully disclosure of all study relevant information with regard to intellectual property protection which makes is very difficult to compare the different trials with each other (Kabat et al., 2020). One route to overcome certain levels of this inconsistent trial outcomes is to set relevant quality parameters for MSCs. The initial MSC minimal criteria for the ISCT were focusing on the stemness ability of MSCs (Levy et al., 2020). The recent position statement on nomenclature also include the tissue origin and the functional capacity which have major influences on the mode of action (Viswanathan et al., 2019). The development of artificial intelligence strategies can support unraveling the so far undetermined factors within MSC-based therapies (Zhou et al., 2021). Prior assessment of MSCs surface marker composition as well as gene signature may 
advance the prediction in therapy outcome and may lead to more effective cell manufacturing processes (Lipsitz et al., 2016). The distinct cell populations applied in the different studies are rarely completely characterized which makes it very challenging to compare the various studies and furthermore to reproduce them (Wilson et al., 2019). Approaches for the development of new protocols for stable MSC propagation in vitro to enable larger yields or the establishment of large MSC banks are promising but need critical evaluation (Lechanteur et al., 2016; Wilson et al., 2019). Potency assays should be carried out to address MSC functionality, the currently accepted assay is the suppression of in vitro T-cell proliferation (de Wolf et al., 2017).

Several biomaterial approaches have been developed with the aim to increase the homogeneity of MSCs during the propagation phase prior to clinical use (Levy et al., 2020). For example, specific biological materials can be used to improve MSCs delivery and survival (Zhou et al., 2021). An example for this is the loading of MSCs with microparticles which encapsulate smallmolecules (Tzouanas et al., 2014) or synthetic polymers as ECMs. Furthermore, gene therapy approaches may lead to improved MSC performance for a particular application and could increase the degree of homogeneity. There is a clinical trial underway to investigate the use of genetically modified MSCs in treating Kabuki Syndrome (NCT03855631) (Zhou et al., 2021). Another way to support MSC treatment efficiency is the priming of MSCs before admission. With this approach, MSCs were shown to be in an exogenously boosted more potent state compared with the unprimed cells (Zhou et al., 2021). Going away from transplanting cells toward transplanting secreted factors such as extracellular vesicles has also shown promising results in trials for GvHD or chronic kidney disease (Lai et al., 2018; Dreyer et al., 2020). Engineering processes for large scale production of MSC derived extracellular vesicles could be a standardized process (Zhou et al., 2021).

\section{iMSCs as a Complementary Cell Source for Therapy \\ Pre-clinical Use of iMSCs}

As described above, there are many substantial issues in the use of native MSCs for therapeutic approaches. Because of these issues, MSCs so far have only been successfully converted into treatment of patients to a certain extent. In contrast to native MSCs with all their restrictions, an unlimited and safe source such as iPSCderived iMSCs would be a great alternative (Zhao and Ikeya, 2018). Although the collection processes of for example bone marrow and the subsequent isolation of MSCs are standardized and carried out in a routine fashion, it is still an invasive process and is associated with donor-site morbidities (Bhumiratana et al., 2016). Furthermore, the cell number for transplantation is a critical point. Since the initial cell number from bone marrow aspirates is restricted, cells have to be expanded in vitro which is associated with the problem of decreased proliferation and differentiation potential upon prolonged in vitro culture (Duscher et al., 2014; Palumbo et al., 2014; Yang et al., 2018). This challenge also could be overcome by the use of iMSCs since iPSCs can be indefinitely expanded and then differentiated in the needed amount of iMSCs. By their ability to indefinite selfrenewal iPSCs are similar to ESCs (Prigione et al., 2011; Drews et al., 2012). The iPSCs can be differentiated into MSCs, the so called iMSCs. These iMSCs show similar characteristics as native MSCs with regard to their immunophenotype, differentiation potential, and secretory profile (Chen et al., 2012; Frobel et al., 2014; Kimbrel et al., 2014; Hu et al., 2015; Spitzhorn et al., 2019). As alternative to the use of primary MSCs the route via iPSCs into iMSCs is promising. Upon the reprogramming process into iPSCs, the cells are shifted into a phenotype which does not show characteristics of an aged cell. Additionally, there are reports that iMSCs are in a rejuvenated state compared to the primary MSCs. Reports have shown that iMSC lose the age-related and tissue-specific DNA methylation profiles but maintain a donor specific DNA methylation signature (Lian et al., 2010). Especially the use of iMSCs from iPSCs which were derived from stem cells which are obtained non-invasively such as urine are a very promising alternative (Rahman et al., 2020). When the initial cell material is chosen wisely, iPSCs can be obtained with non- or minimal invasive procedures and they are ethical unproblematic. When used autologous or with matching HLA type the frequency of unwanted immune reactions is diminished (de Rham and Villard, 2014; Bohndorf et al., 2017). Several protocols have been developed to generate iMSCs from iPSCs including the use of FGF supplementation or the inhibition of TGFb signaling (Zhao and Ikeya, 2018). As mentioned above most MSCs are derived from bone marrow or other tissues. Since invasive procedures are necessary, the donor is faced with several risks (Sheyn et al., 2016). Since iPSCs can be expanded limitless, this broad available starting material has the potential to increase the homogeneity and standardized procedure in iMSC use.

iMSCs have been used in various in vivo models for multiple sclerosis, limb ischemia, autoimmunity, hypoxic ischemia and autosomal inherited liver disease and bone defect healing (Lian et al., 2010; Gruenloh et al., 2011; Kimbrel et al., 2014; Wang et al., 2014; Hawkins et al., 2018; Spitzhorn et al., 2018; Jungbluth et al., 2019). Interestingly, it has been reported that iMSCs were even able to outperform their native counterparts (Wang et al., 2014; Hawkins et al., 2018), particularly concerning the release of exosomes and support of mitochondrial transfer function (Li et al., 2014; Zhao and Ikeya, 2018). Zhang et al. (2016) and Yang et al. (2020) report mitochondrial transfer in iMSCs co-cultured with rodent cellular models of induced neuronal and cardiac injury. The mitochondrial transfer is accomplished by nanotubules. Zhang et al. (2016) suggest that higher expression of Rho GTPase 1 (MIRO1) and TNFaIP2 leads to superior mitochondrial transfer in iMSCs compared to BMMSCs via tumor-necrosis-factor-alpha-(TNF)-induced tunneling nanotube formation. Although they are derived from potential tumorigenic pluripotent cells, none of the previous mentioned studies have reported tumor formation of the iMSCs and in a liver regeneration study they did not cause any signs of tumor formation after 2 months (Spitzhorn et al., 2018). The generation and propagation of iPSC-derived MSCs was shown to be applicable to GMP conditions in a routine manner (Ozay et al., 2019). Thawed iMSCs had a low degree of senescence 


\section{Terminally differentiated cells}
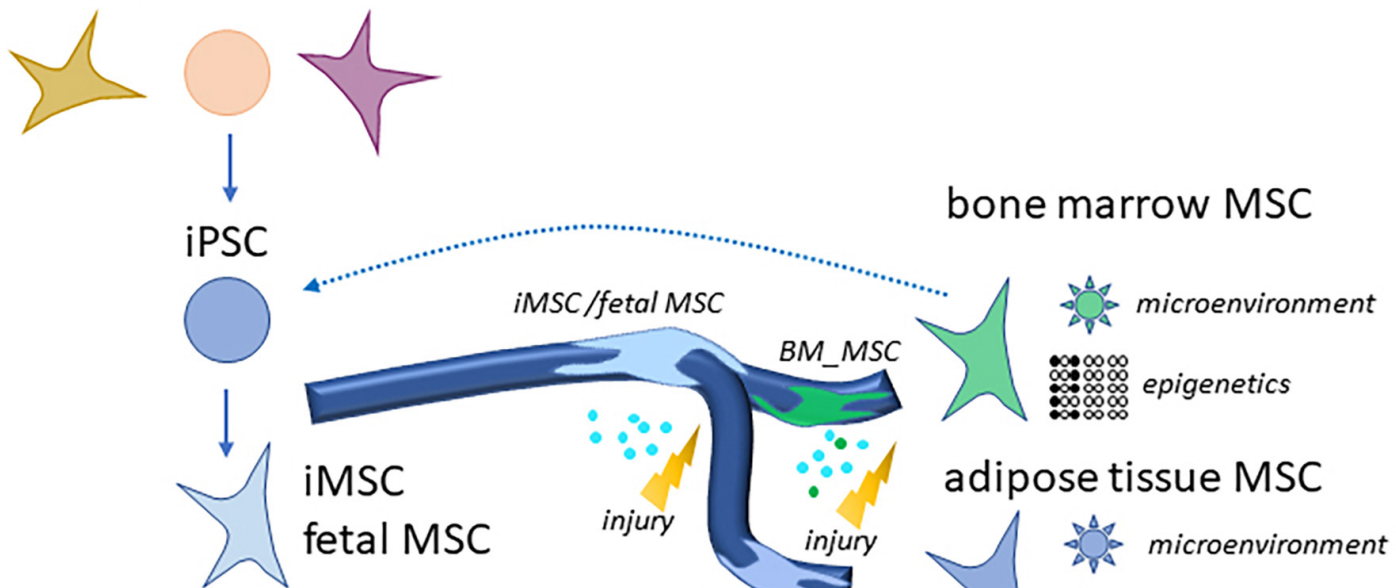

epigenetics

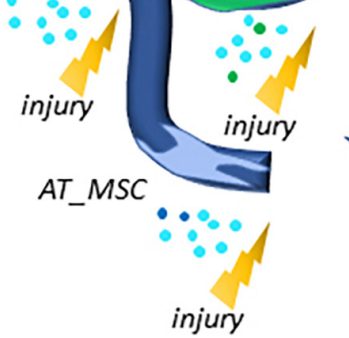

adipose tissue MSC

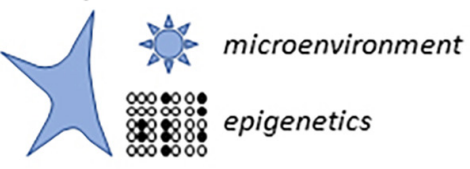

FIGURE 7 | Generation of rejuvenated and more homogenous iMSCs via reprogramming of MSCs or terminally differentiated cells to iPSCs. Distinct MSC subpopulations such as bone marrow MSCs and adipose tissue MSCs have high levels of heterogeneity due to their microenvironments and epigenetic programs. This gets more similar as well as rejuvenated in iMSCs. MSCs as well as iMSCs home into sites of injury and exert beneficial effects which are predominantly due to paracrine signaling and are even superior for the iMSCs as several studies demonstrated.

and were immunomodulatory active by IDO mediated immune suppression (Chinnadurai et al., 2017).

\section{Clinical Trials Employing iMSCs}

Another re-assuring evidence in support of the use of the iMSC concept in regenerative medicine and cell replacement therapy is their use in clinical trials. Up until now, iMSCs have been implemented in six clinical trials. Cynata Therapeutics Ltd. (Australia) has finished a phase I clinical trial investigating the safety, tolerability and efficacy using iMSCs which were derived from iPSCs treating steroid resistant acute GvHD in adults with preliminary promising results (NCT02923375). The company furthermore is just recruiting patients for a study to examine the early efficacy of intravenous administration of iMSCs derived from iPSCs in adults which have been brought to an intensive care unit with COVID-19 infection (NCT04537351). In a second phase of this study the effect of iMSCs on the Acute Respiratory Distress Syndrome will be assessed. The company has now announced a Phase 3 clinical trial of CYP-004, its Cymerus mesenchymal stem cell (MSC) product for osteoarthritis. Another study which is performed by the Tongji Hospital (China) in cooperation with the Chinese Academy of Sciences is investigating the safety of ESC-derived iMSCs in treating meniscus injury patients in a phase I clinical trial (NCT03839238). The Department of Urology of the Asan Medical Center in Seoul, South Korea is running a Phase 1 Study to investigate the safety of human ESC-derived iMSCs in the treatment of Interstitial Cystitis (NCT04610359). Another Phase 1 safety study is carried out by The First Affiliated Hospital of Zhengzhou
University in China. Aim of this study is to evaluate the safety of ESC-derived iMSC for the treatment of Primary Ovarian Insufficiency (NCT03877471). The Tongji Hospital in China is evaluating the safe clinical use of intrauterine injected human ESC-derived iMSCs to treat moderate and severe intrauterine adhesion. In addition to safety this study should bring first insights for clinical effectiveness in supporting the regeneration and repair process within the endometrial region (NCT04232592) $)^{1,2}$.

The pioneer study from Cynata Therapeutics Ltd., of iMSCs in the treatment of GvHD (NCT02923375) is the first completed clinical trial for the use of iMSCs (Bloor et al., 2020). The clinical data confirmed the safety and tolerability of their iMSC product. However, further studies on more than 15 probands and for efficacy testing have to be carried out (Bloor et al., 2020). In this study the authors describe the three-staged generation process in detail. In a first step, the iPSCs are banked and expanded. The iPSCs which were used in the study were generated by Cellular Dynamics International, Inc., from mononuclear blood cells of a healthy adult donor using episomal-based nonintegrating reprogramming method oriP/EBNA1-based plasmids (Mack et al., 2011; Bloor et al., 2020). Subsequently, the iPSCs are differentiated into iMSCs in the second step. The final step is the iMSCs expansion and the implementation of the final medicinal product. With this process, according to the authors' calculations, it is possible to derive $9 \times 10^{4}$ vials, each containing $1 \times 10^{6}$ iPSCs which in the end iMSCs stage have reached a number of $2.9 \times 10^{15}$ iMSCs which represent 29 million therapeutic doses

\footnotetext{
${ }^{2}$ https://hpscreg.eu/
} 
of $1 \times 10^{8}$ cells- all started with iPSC generation from one single blood donation (Bloor et al., 2020). iPSC quality control includes PCR, comparative genomic hybridization and singlenucleotide polymorphism analysis. Since the middle stage the iPSCs are tumorigenic by nature the manufacturer has to ensure the complete absence of undifferentiated iPSCs in the end stage of iMSCs. Therefore, several screening steps are carried out which include medium selection which does not support iPSC growth, physical separation of undifferentiated cell clusters and culture conditions that will favor iMSC growth such as plastic adherence. Furthermore, qPCR and in vitro tumorigenicity assays were done. This process is verified by using undifferentiated iPSCs which led to the result that no iPSC colony was detectable thereafter. The iMSCs were characterized by cell surface marker expression CD105+, CD73+, CD90+, CD43/45-, CD31-, and HLA-DR-. A high degree of inter-LOT consistency was claimed based on transcriptome analysis (Bloor et al., 2020). Based on the maximal used dose of $2 \times 10^{8}$ cells per $\mathrm{kg}$ body weight which were administered intravenously on Day 0 and Day 7, the authors calculated that a single iPSC Bank would be able to produce 29 million clinical doses of the iMSC product (Bloor et al., 2020). These production capacities are of course highly connected to the capacities of the iPSC bank. By using the iPSC to iMSCs approach the cellular expansion can be shifted from the MSC to the iPSC stage and enable expansion to large quantities with the same starting material (Ozay et al., 2019). The iMSC product has already been applied in other diseases on a pre-clinical level such as asthma, limb ischemia, or rejection of organs (Bloor et al., 2020). The route via PSCs makes those cells also eligible for gene editing technologies such as CRISPR/Cas9 and gene-directed enzyme prodrug therapy (Doudna and Charpentier, 2014).

\section{General Outlook}

iMSCs as an off-the shelf product with high batch to batch consistency will result in more robust clinical measures (Wilson et al., 2019). Furthermore, iMSCs could serve as a reference material in the research environment to ensure MSC as well as iMSCs standards worldwide. Cell based reference materials (reference cell line) which are available to all laboratories could help in optimizing clinical outcomes and mastering MSC-associated (heterogeneity) challenges (Viswanathan et al., 2014). Manufacturing of such a line is challenging by having large quantities of cells, and stably expandable cells with MSC properties, iMSCs may be of use for this case (Tanavde et al., 2015). Until the use of iMSCs becomes routine in clinical studies critical issues such as optimized generation protocols, deciphering their mode of action and differentiation routes have to be fully understood to ensure higher degree of clinical efficiency and safe use (Zhao and Ikeya, 2018).

\section{DISCUSSION}

In this study on the heterogeneity of MSCs, we comparatively characterized the transcriptomes of MSC subpopulations and the relationships amongst them. We found large commonalities between them but also differences related to the tissue source of the MSCs. In a hierarchical clustering analysis we could stratify the analyzed stem cells into three meta-groups containing PSCs (embryonal and induced), MSCs of adult origin of various tissues and MSCs of young origin (fetal, UC, and iMSCs). Comparing the expressed genes (detection- $p$-value $<0.05$ ) between iMSCs fetal, MSCs, UC-MSCs, BM-MSCs, and ATMSCs we found most genes $(n=9966)$ overlapping with smaller specific subsets of exclusively expressed genes ( $n$ between 29 and 558). Over-represented GOs in the exclusive subsets related to developmental processes in the young MSCs, to adipose-tissuerelated (vascular, muscle, and neuronal development) in ATMSCs and bone-related (skeletal system and limb development) in BM-MSCs. We extended the comparison to MSCs of other distinct sources (AF-MSCs, UdRPCs, and HSCs) generated on different technical platforms and again found the most genes in the overlap $(n=6637)$ and fewer genes in the exclusive subsets (between 301 and 2,280). Here, over-represented GOs in the exclusive subsets related to developmental processes in the AF-MSCs which may be assigned to the young metagroup, over-represented GO terms in UdRPCs included lipid transport and detoxification pointing at renal properties and over-represented GO terms in HSCs included metabolic, cristae, and mitochondrial processes characterizing liver.

We further investigated if we could use the iMSC concept to revert adult cells from heterogeneous populations to a defined MSC initial state and found evidence for that in overlapping developmental biological processes (GOs) with the other young MSCs (fetal and UC-MSCs) and by clustering with the other young MSCs in heatmaps using our previous aging-rejuvenation gene signature (Spitzhorn et al., 2019). These analyses showed that gene-regulatory networks driving rejuvenation are activated in iMSCs. To refine this search to epigenetic causes we performed hierarchical clustering with a gene signature related to epigenetic mechanisms described by Avgustinova and Benitah (2016) and also with this signature we could get defined clusters of PSCs, adult MSCs and marginally fragmented young MSCs. Although this is no direct epigenetic analysis it demonstrates that epigenetic mechanisms connected to the expression of this gene signature are active in the differentiation and rejuvenation of these MSCs. The causal relationship between aging and epigenetic changes has been demonstrated by Horvath's epigenetic clock enabling prediction of the age based on an individual's methylation profile (Horvath, 2013). While the aging-related long-term changes manifest more prominently on the methylome but on the other hand work through effects on the transcriptome we could also identify a rejuvenation signature in MSCs on the transcriptome level (Spitzhorn et al., 2019). Distinct lineages and states of cellular differentiation alongside aging are associated with pronounced epigenetics programs in regulating MSC homeostasis (Sui et al., 2020).

We propose that the source of the MSCs characterizes the subpopulation by exchanging signals with the microenvironment and by epigenetic programs. We found that iMSCs derived from iPSCs resemble other MSCs of young age such as fetal MSCs and UC-MSCs and are determined by developmental biological processes. That confirms our earlier findings (Spitzhorn et al., 2019) and may imply that the epigenetic programs of MSCs 
in differentiated and aged microenvironments such as bone marrow or adipose tissue can be reset to an initial state as shown in Figure 7. Also Frobel et al. (2014) report an epigenetic rejuvenation of age- and tissue-related DNA methylation in iPSC-derived MSCs while retaining donor-specific methylation patterns. This initial epigenetic state represented in the iMSCs could provide a means of overcoming heterogeneity and thus a reproducible standard for therapies.

An advantage of MSCs is their more differentiated state compared to PSCs what was considered to protect them from tumorigenicity (Diederichs and Tuan, 2014). However, in recent literature the image of a double-edged sword came up meaning that MSCs can promote and inhibit cancer (Lee and Hong, 2017; Galland and Stamenkovic, 2020). In that context CSCs which are defined rather by CD133 than MSC marker expression have been described to arise from epithelial-mesenchymal-transition (EMT) of cancer cells (Shibue and Weinberg, 2017). This suggests plasticity of these mesenchymal cell types and may raise questions by which changes to the microenvironment and epigenetics they are transformable into each other. Transitions between these cell states may putatively be connected to cancerogenesis or on the other hand have the potential to drive cancer cells to a differentiated state. Thus, their study may elucidate if and in what microenvironment MSCs have tumor-promoting or inhibiting effects (Diederichs and Tuan, 2014; Papaccio et al., 2017; Galland and Stamenkovic, 2020) with the aim to find the best therapeutic states.

In conclusion, in this review and meta-analysis comparing MSCs from multiple tissues and donor ages we have found predominant commonalities but also differences contributing to heterogeneity. Differences between subpopulations were related to the tissue source environment which also holds for MSC cells such as UdRPCs showing renal properties and HSCs showing hepatic properties but still bearing more commonalities than differences to other MSC types. iMSCs, MSCs derived from iPSCs, have overlapping developmental biological processes with other young MSCs of fetal or umbilical cord origin and thus confirmed our previous rejuvenation signature (Spitzhorn et al.,

\section{REFERENCES}

Aliperta, R., Cartellieri, M., Feldmann, A., Arndt, C., Koristka, S., Michalk, I., et al. (2015). Bispecific antibody releasing-mesenchymal stromal cell machinery for retargeting T cells towards acute myeloid leukemia blasts. Blood Cancer J. 5:e348. doi: 10.1038/bcj.2015.73

Almeida-Porada, G., Atala, A. J., and Porada, C. D. (2020). Therapeutic mesenchymal stromal cells for immunotherapy and for gene and drug delivery. Mol. The. Methods Clin. Dev. 16, 204-224. doi: 10.1016/j.omtm.2020.01.005

Avgustinova, A., and Benitah, S. A. (2016). Epigenetic control of adult stem cell function. Nat. Rev. Mol. Cell Biol. 17, 643-658. doi: 10.1038/nrm.2016.76

Barberi, T., Willis, L. M., Socci, N. D., and Studer, L. (2005). Derivation of multipotent mesenchymal precursors from human embryonic stem cells. PLoS Med. 2:e161. doi: 10.1371/journal.pmed.0020161

Barrett, A. N., Fong, C.-Y., Subramanian, A., Liu, W., Feng, Y., Choolani, M., et al. (2019). Human Wharton's Jelly mesenchymal stem cells show unique gene expression compared with bone marrow mesenchymal stem cells using Single-Cell RNA-Sequencing. Stem Cells Dev. 28, 196-211. doi: 10.1089/scd. 2018.0132
2019). We demonstrated that rejuvenation can be driven by epigenetic mechanisms and propose that heterogeneity resulting from distinct microenvironmental stimuli and epigenetic patterns may be reset to a rejuvenated state via cellular reprogramming and differentiation into iMSCs.

\section{AUTHOR CONTRIBUTIONS}

WW, NG, L-SS, and JA wrote the manuscript. WW performed the meta-analysis and follow-up analyses. JA initiated and supervised the study. All authors contributed to the article and approved the submitted version.

\section{FUNDING}

JA acknowledges the Medical Faculty of Heinrich Heine University Düsseldorf for financial support.

\section{SUPPLEMENTARY MATERIAL}

The Supplementary Material for this article can be found online at: https://www.frontiersin.org/articles/10.3389/fcell.2021. 717772/full\#supplementary-material

Supplementary Table 1 | Subsets and GO analyses of relevant subsets of the venn diagram of fetal MSCs, iMSCs, adipose-tissue-MSCs, bone-marrow-MSCs and UC-MSCs (Figures 2 and $\mathbf{3}$ ). (A) subsets of genes, (B) Significant GO terms in fetal MSCs (401 genes), (C) Significant GO terms in UC-MSCs (558 genes), (D) Significant GO terms in iMSCs (374 genes), (E) Significant GO terms in adipose-tissue-derived MSCs (29 genes), (F) Significant GO terms in bone-marrow-derived MSCs (130 genes), (G) Significant GO terms in the intersection of all (9966 genes).

Supplementary Table 2 | Subsets and GO analyses of relevant subsets of the venn diagram of amniotic-fluid-derived MSCs (AF-MSCs), urine-derived renal progenitor cells (UdRPCs) and hepatic stellate cells (HSCs) and the common MSC signature determined before (Figure 4). (A) subsets of genes, (B) Significant GO terms in AF-MSCs (301 genes), (C) Significant GO terms in UdRPCs (476 genes), (D) Significant GO terms in HSCs (2280 genes).
Bernstein, B. E., Meissner, A., and Lander, E. S. (2007). The mammalian epigenome. Cell 128, 669-681. doi: 10.1016/j.cell.2007.01.033

Bhumiratana, S., Bernhard, J. C., Alfi, D. M., Yeager, K., Eton, R. E., Bova, J., et al. (2016). Tissue-engineered autologous grafts for facial bone reconstruction. Sci. Transl. Med. 8:343ra83. doi: 10.1126/scitranslmed.aad5904

Bloor, A. J. C., Patel, A., Griffin, J. E., Gilleece, M. H., Radia, R., Yeung, D. T., et al. (2020). Production, safety and efficacy of iPSC-derived mesenchymal stromal cells in acute steroid-resistant graft versus host disease: a phase I, multicenter, open-label, dose-escalation study. Nat. Med. 26, 1720-1725. doi: 10.1038/s41591-020-1050-x

Bohndorf, M., Ncube, A., Spitzhorn, L.-S., Enczmann, J., Wruck, W., and Adjaye, J. (2017). Derivation and characterization of integration-free iPSC line ISRMUM51 derived from SIX2-positive renal cells isolated from urine of an African male expressing the CYP2D6 $* 4 /{ }^{*} 17$ variant which confers intermediate drug metabolizing activity. Stem Cell Res. 25, 18-21. doi: 10.1016/j.scr.2017. 10.004

Boyle, A. J., Schulman, S. P., and Hare, J. M. (2006). Stem cell therapy for cardiac repair: ready for the next step. Circulation 114, 339-352. doi: 10.1161/ CIRCULATIONAHA.105.590653 
Čamernik, K., Mihelič, A., Mihalič, R., Haring, G., Herman, S., Marolt Presen, D., et al. (2020). Comprehensive analysis of skeletal muscle- and bone-derived mesenchymal stem/stromal cells in patients with osteoarthritis and femoral neck fracture. Stem Cell Res. Ther. 11:146. doi: 10.1186/s13287-020-01657-z

Caplan, A. I. (1991). Mesenchymal stem cells. J. Orthop. Res. 9, 641-650. doi: 10.1002/jor.1100090504

Caplan, A. I. (2008). All MSCs are pericytes? Cell Stem Cell 3, 229-230. doi: 10.1016/j.stem.2008.08.008

Caplan, A. I. (2017). Mesenchymal stem cells: time to change the name!: mesenchymal stem cells. Stem Cells Transl. Med. 6, 1445-1451. doi: 10.1002/ sctm.17-0051

Caplan, A. I., and Correa, D. (2011). The MSC: an injury drugstore. Cell Stem Cell 9, 11-15. doi: 10.1016/j.stem.2011.06.008

Carter, B., and Zhao, K. (2020). The epigenetic basis of cellular heterogeneity. Nat. Rev. Genet. 22, 235-250. doi: 10.1038/s41576-020-00300-0

Carvalho, A. É. S., Sousa, M. R. R., Alencar-Silva, T., Carvalho, J. L., and SaldanhaAraujo, F. (2019). Mesenchymal stem cells immunomodulation: the road to IFN- $\gamma$ licensing and the path ahead. Cytokine Growth Factor Rev. 47, 32-42. doi: 10.1016/j.cytogfr.2019.05.006

Chen, P.-M., Yen, M.-L., Liu, K.-J., Sytwu, H.-K., and Yen, B.-L. (2011). Immunomodulatory properties of human adult and fetal multipotent mesenchymal stem cells. J. Biomed. Sci. 18:49. doi: 10.1186/1423-0127-18-49

Chen, Q., Shou, P., Zheng, C., Jiang, M., Cao, G., Yang, Q., et al. (2016). Fate decision of mesenchymal stem cells: adipocytes or osteoblasts? Cell Death Differ. 23, 1128-1139. doi: 10.1038/cdd.2015.168

Chen, Y. S., Pelekanos, R. A., Ellis, R. L., Horne, R., Wolvetang, E. J., and Fisk, N. M. (2012). Small molecule mesengenic induction of human induced pluripotent stem cells to generate mesenchymal stem/stromal cells. Stem Cells Transl. Med. 1, 83-95. doi: 10.5966/sctm.2011-0022

Chen, Y.-H., Chung, C.-C., Liu, Y.-C., Yeh, S.-P., Hsu, J. L., Hung, M.C., et al. (2016). Enhancer of zeste homolog 2 and histone deacetylase 9c Regulate age-dependent mesenchymal stem cell differentiation into osteoblasts and adipocytes. Stem Cells 34, 2183-2193. doi: 10.1002/stem. 2400

Chinnadurai, R., Rajan, D., Ng, S., McCullough, K., Arafat, D., Waller, E. K., et al. (2017). Immune dysfunctionality of replicative senescent mesenchymal stromal cells is corrected by IFN $\gamma$ priming. Blood Adv. 1, 628-643. doi: 10.1182/ bloodadvances. 2017006205

Cho, J., D’Antuono, M., Glicksman, M., Wang, J., and Jonklaas, J. (2018). A review of clinical trials: mesenchymal stem cell transplant therapy in type 1 and type 2 diabetes mellitus. Am. J. Stem Cells 7, 82-93.

Conrad, C., Niess, H., Huss, R., Huber, S., von Luettichau, I., Nelson, P. J., et al. (2009). Multipotent mesenchymal stem cells acquire a lymphendothelial phenotype and enhance lymphatic regeneration in vivo. Circulation 119, 281289. doi: 10.1161/CIRCULATIONAHA.108.793208

Costa, L. A., Eiro, N., Fraile, M., Gonzalez, L. O., Saá, J., Garcia-Portabella, P., et al. (2021). Functional heterogeneity of mesenchymal stem cells from natural niches to culture conditions: implications for further clinical uses. Cell. Mol. Life Sci. 78, 447-467. doi: 10.1007/s00018-020-03600-0

Crisan, M., Yap, S., Casteilla, L., Chen, C.-W., Corselli, M., Park, T. S., et al. (2008). A perivascular origin for mesenchymal stem cells in multiple human organs. Cell Stem Cell 3, 301-313. doi: 10.1016/j.stem.2008.07.003

de Almeida, D. C., Ferreira, M. R. P., Franzen, J., Weidner, C. I., Frobel, J., Zenke, M., et al. (2016). Epigenetic classification of human mesenchymal stromal cells. Stem Cell Rep. 6, 168-175. doi: 10.1016/j.stemcr.2016.01.003

de Rham, C., and Villard, J. (2014). Potential and limitation of HLA-based banking of human pluripotent stem cells for cell therapy. J. Immunol. Res. 2014:518135. doi: $10.1155 / 2014 / 518135$

de Wolf, C., van de Bovenkamp, M., and Hoefnagel, M. (2017). Regulatory perspective on in vitro potency assays for human mesenchymal stromal cells used in immunotherapy. Cytotherapy 19, 784-797. doi: 10.1016/j.jcyt.2017.03. 076

Diederichs, S., and Tuan, R. S. (2014). Functional comparison of human-induced pluripotent stem cell-derived mesenchymal cells and bone marrow-derived mesenchymal stromal cells from the same donor. Stem Cells Dev. 23, 1594-1610. doi: $10.1089 /$ scd.2013.0477

Dominici, M., Le Blanc, K., Mueller, I., Slaper-Cortenbach, I., Marini, F., Krause, D., et al. (2006). Minimal criteria for defining multipotent mesenchymal stromal cells. The international society for cellular therapy position statement. Cytotherapy 8, 315-317. doi: 10.1080/14653240600855905

Doudna, J. A., and Charpentier, E. (2014). Genome editing. The new frontier of genome engineering with CRISPR-Cas9. Science 346:1258096. doi: 10.1126/ science. 1258096

Drews, K., Jozefczuk, J., Prigione, A., and Adjaye, J. (2012). Human induced pluripotent stem cells-from mechanisms to clinical applications. J. Mol. Med. (Berl.) 90, 735-745. doi: 10.1007/s00109-012-0913-0

Dreyer, G. J., Groeneweg, K. E., Heidt, S., Roelen, D. L., van Pel, M., Roelofs, H., et al. (2020). Human leukocyte antigen selected allogeneic mesenchymal stromal cell therapy in renal transplantation: the Neptune study, a phase I single-center study. Am. J. Transplant. 20, 2905-2915. doi: 10.1111/ajt.15910

Driscoll, J., and Patel, T. (2019). The mesenchymal stem cell secretome as an acellular regenerative therapy for liver disease. J. Gastroenterol. 54, 763-773. doi: 10.1007/s00535-019-01599-1

Du, P., Kibbe, W. A., and Lin, S. M. (2008). lumi: a pipeline for processing Illumina microarray. Bioinformatics 24, 1547-1548. doi: 10.1093/bioinformatics/btn224

Duscher, D., Rennert, R. C., Januszyk, M., Anghel, E., Maan, Z. N., Whittam, A. J., et al. (2014). Aging disrupts cell subpopulation dynamics and diminishes the function of mesenchymal stem cells. Sci. Rep. 4:7144. doi: 10.1038/srep07144

Falcon, S., and Gentleman, R. (2007). Using GOstats to test gene lists for GO term association. Bioinformatics 23, 257-258. doi: 10.1093/bioinformatics/btl567

Fernández, A. F., Bayón, G. F., Urdinguio, R. G., Toraño, E. G., García, M. G., Carella, A., et al. (2015). H3K4mel marks DNA regions hypomethylated during aging in human stem and differentiated cells. Genome Res. 25, 27-40. doi: 10.1101/gr.169011.113

Fitzsimmons, R. E. B., Mazurek, M. S., Soos, A., and Simmons, C. A. (2018). Mesenchymal Stromal/stem cells in regenerative medicine and tissue engineering. Stem Cells Int. 2018, 8031718. doi: 10.1155/2018/8031718

Friedenstein, A. J., Chailakhjan, R. K., and Lalykina, K. S. (1970). The development of fibroblast colonies in monolayer cultures of guinea-pig bone marrow and spleen cells. Cell Tissue Kinet. 3, 393-403. doi: 10.1111/j.1365-2184.1970. tb00347.x

Frobel, J., Hemeda, H., Lenz, M., Abagnale, G., Joussen, S., Denecke, B., et al. (2014). Epigenetic rejuvenation of mesenchymal stromal cells derived from induced pluripotent stem cells. Stem Cell Rep. 3, 414-422. doi: 10.1016/j.stemcr. 2014.07.003

Galili, T. (2015). dendextend: an R package for visualizing, adjusting and comparing trees of hierarchical clustering. Bioinformatics 31, 3718-3720. doi: 10.1093/bioinformatics/btv428

Galipeau, J., and Sensébé, L. (2018). Mesenchymal stromal cells: clinical challenges and therapeutic opportunities. Cell Stem Cell 22, 824-833. doi: 10.1016/j.stem. 2018.05.004

Galland, S., and Stamenkovic, I. (2020). Mesenchymal stromal cells in cancer: a review of their immunomodulatory functions and dual effects on tumor progression. J. Pathol. 250, 555-572. doi: 10.1002/path.5357

Ganguly, P., El-Jawhari, J. J., Giannoudis, P. V., Burska, A. N., Ponchel, F., and Jones, E. A. (2017). Age-related changes in bone marrow mesenchymal stromal cells: a potential impact on osteoporosis and osteoarthritis development. Cell Transplant. 26, 1520-1529. doi: 10.1177/0963689717721201

Gardiner-Garden, M., and Frommer, M. (1987). CpG islands in vertebrate genomes. J. Mol. Biol. 196, 261-282. doi: 10.1016/0022-2836(87)90689-9

Gautier, L., Cope, L., Bolstad, B. M., and Irizarry, R. A. (2004). affy-analysis of Affymetrix GeneChip data at the probe level. Bioinformatics 20, 307-315. doi: 10.1093/bioinformatics/btg405

Gentleman, R. C., Carey, V. J., Bates, D. M., Bolstad, B., Dettling, M., Dudoit, S., et al. (2004). Bioconductor: open software development for computational biology and bioinformatics. Genome Biol. 5:R80. doi: 10.1186/gb-2004-5-10r80

Graffmann, N., Ring, S., Kawala, M.-A., Wruck, W., Ncube, A., Trompeter, H.-I., et al. (2016). Modeling nonalcoholic fatty liver disease with human pluripotent stem cell-derived immature hepatocyte-like cells reveals activation of PLIN2 and confirms regulatory functions of peroxisome proliferatoractivated receptor alpha. Stem Cells Dev. 25, 1119-1133. doi: 10.1089/scd.2015. 0383

Gressner, A. M., and Weiskirchen, R. (2006). Modern pathogenetic concepts of liver fibrosis suggest stellate cells and TGF-beta as major players and therapeutic targets. J. Cell. Mol. Med. 10, 76-99. 
Gruenloh, W., Kambal, A., Sondergaard, C., McGee, J., Nacey, C., Kalomoiris, S., et al. (2011). Characterization and in vivo testing of mesenchymal stem cells derived from human embryonic stem cells. Tissue Eng. Part A 17, 1517-1525. doi: 10.1089/ten.TEA.2010.0460

Haga, H., Yan, I. K., Takahashi, K., Wood, J., Zubair, A., and Patel, T. (2015). Tumour cell-derived extracellular vesicles interact with mesenchymal stem cells to modulate the microenvironment and enhance cholangiocarcinoma growth. J. Extracell Vesicles 4:24900. doi: 10.3402/jev.v4.24900

Haider, S., Ballester, B., Smedley, D., Zhang, J., Rice, P., and Kasprzyk, A. (2009). BioMart Central Portal-unified access to biological data. Nucleic Acids Res. 37, W23-W27. doi: 10.1093/nar/gkp265

Harrell, C. R., Jovicic, N., Djonov, V., Arsenijevic, N., and Volarevic, V. (2019). Mesenchymal stem cell-derived exosomes and other extracellular vesicles as new remedies in the therapy of inflammatory diseases. Cells 8:1605. doi: 10 . $3390 /$ cells 8121605

Hawkins, K. E., Corcelli, M., Dowding, K., Ranzoni, A. M., Vlahova, F., Hau, K.-L., et al. (2018). Embryonic stem cell-derived mesenchymal stem cells (MSCs) have a superior neuroprotective capacity over fetal MSCs in the hypoxic-ischemic mouse brain. Stem Cells Transl. Med. 7, 439-449. doi: 10.1002/sctm.17-0260

Hemming, S., Cakouros, D., Isenmann, S., Cooper, L., Menicanin, D., Zannettino, A., et al. (2014). EZH2 and KDM6A act as an epigenetic switch to regulate mesenchymal stem cell lineage specification. Stem Cells 32, 802-815. doi: 10. 1002/stem.1573

Horvath, S. (2013). DNA methylation age of human tissues and cell types. Genome Biol. 14:R115. doi: 10.1186/gb-2013-14-10-r115

Horwitz, E. M., Le Blanc, K., Dominici, M., Mueller, I., Slaper-Cortenbach, I., Marini, F. C., et al. (2005). Clarification of the nomenclature for MSC: the international society for cellular therapy position statement. Cytotherapy 7 , 393-395. doi: 10.1080/14653240500319234

Hu, G., Li, Q., Niu, X., Hu, B., Liu, J., Zhou, S., et al. (2015). Exosomes secreted by human-induced pluripotent stem cell-derived mesenchymal stem cells attenuate limb ischemia by promoting angiogenesis in mice. Stem Cell Res. Ther. 6:10. doi: 10.1186/scrt546

Huang, Y., Li, Q., Zhang, K., Hu, M., Wang, Y., Du, L., et al. (2019). Single cell transcriptomic analysis of human mesenchymal stem cells reveals limited heterogeneity. Cell Death Dis. 10:368. doi: 10.1038/s41419-0191583-4

Jing, H., Liao, L., An, Y., Su, X., Liu, S., Shuai, Y., et al. (2016). Suppression of $\mathrm{EZH} 2$ prevents the shift of osteoporotic MSC fate to adipocyte and enhances bone formation during osteoporosis. Mol. Ther. 24, 217-229. doi: 10.1038/mt. 2015.152

Johnson, K., Zhu, S., Tremblay, M. S., Payette, J. N., Wang, J., Bouchez, L. C., et al. (2012). A stem cell-based approach to cartilage repair. Science 336, 717-721. doi: 10.1126/science.1215157

Jung, Y.-D., Park, S.-K., Kang, D., Hwang, S., Kang, M.-H., Hong, S.-W., et al. (2020). Epigenetic regulation of miR-29a/miR-30c/DNMT3A axis controls SOD2 and mitochondrial oxidative stress in human mesenchymal stem cells. Redox Biol. 37:101716. doi: 10.1016/j.redox.2020.101716

Jungbluth, P., Spitzhorn, L.-S., Grassmann, J., Tanner, S., Latz, D., Rahman, M. S., et al. (2019). Human iPSC-derived iMSCs improve bone regeneration in mini-pigs. Bone Res. 7:32. doi: 10.1038/s41413-0190069-4

Kabat, M., Bobkov, I., Kumar, S., and Grumet, M. (2020). Trends in mesenchymal stem cell clinical trials 2004-2018: is efficacy optimal in a narrow dose range? Stem Cells Transl. Med. 9, 17-27. doi: 10.1002/sctm.19-0202

Keating, A. (2012). Mesenchymal stromal cells: new directions. Cell Stem Cell 10, 709-716. doi: 10.1016/j.stem.2012.05.015

Ketterl, N., Brachtl, G., Schuh, C., Bieback, K., Schallmoser, K., Reinisch, A., et al. (2015). A robust potency assay highlights significant donor variation of human mesenchymal stem/progenitor cell immune modulatory capacity and extended radio-resistance. Stem Cell Res. Ther. 6:236. doi: 10.1186/s13287-0150233-8

Khanh, V. C., Yamashita, T., Ohneda, K., Tokunaga, C., Kato, H., Osaka, M., et al. (2020). Rejuvenation of mesenchymal stem cells by extracellular vesicles inhibits the elevation of reactive oxygen species. Sci. Rep. 10:17315. doi: 10.1038/ s41598-020-74444-8

Kimbrel, E. A., Kouris, N. A., Yavanian, G. J., Chu, J., Qin, Y., Chan, A., et al. (2014). Mesenchymal stem cell population derived from human pluripotent stem cells displays potent immunomodulatory and therapeutic properties. Stem Cells Dev. 23, 1611-1624. doi: 10.1089/scd.2013.0554

Kordes, C., Sawitza, I., Götze, S., Herebian, D., and Häussinger, D. (2014). Stellate cells are mesenchymal stem cells. Eur. J. Med. Res. 19:S6. doi: 10.1186/2047783X-19-S1-S6

Kusuma, G. D., Carthew, J., Lim, R., and Frith, J. E. (2017). Effect of the microenvironment on mesenchymal stem cell paracrine signaling: opportunities to engineer the therapeutic effect. Stem Cells Dev. 26, 617-631. doi: $10.1089 / \mathrm{scd} .2016 .0349$

Lai, P., Chen, X., Guo, L., Wang, Y., Liu, X., Liu, Y., et al. (2018). A potent immunomodulatory role of exosomes derived from mesenchymal stromal cells in preventing cGVHD. J. Hematol. Oncol. 11:135. doi: 10.1186/s13045-0180680-7

Lazarus, H. M., Haynesworth, S. E., Gerson, S. L., Rosenthal, N. S., and Caplan, A. I. (1995). Ex vivo expansion and subsequent infusion of human bone marrowderived stromal progenitor cells (mesenchymal progenitor cells): implications for therapeutic use. Bone Marrow Transplant. 16, 557-564.

Le Blanc, K., Frassoni, F., Ball, L., Locatelli, F., Roelofs, H., Lewis, I., et al. (2008). Mesenchymal stem cells for treatment of steroid-resistant, severe, acute graftversus-host disease: a phase II study. Lancet 371, 1579-1586. doi: 10.1016/ S0140-6736(08)60690-X

Lechanteur, C., Briquet, A., Giet, O., Delloye, O., Baudoux, E., and Beguin, Y. (2016). Clinical-scale expansion of mesenchymal stromal cells: a large banking experience. J. Transl. Med. 14:145. doi: 10.1186/s12967-016-0892-y

Lee, D.-H., Ng, J., Kim, S.-B., Sonn, C. H., Lee, K.-M., and Han, S.-B. (2015). Effect of donor age on the proportion of mesenchymal stem cells derived from anterior cruciate ligaments. PLoS One 10:e0117224. doi: 10.1371/journal.pone. 0117224

Lee, H.-Y., and Hong, I.-S. (2017). Double-edged sword of mesenchymal stem cells: cancer-promoting versus therapeutic potential. Cancer Sci. 108, 1939-1946. doi: 10.1111/cas.13334

Lee, R. H., Kim, B., Choi, I., Kim, H., Choi, H. S., Suh, K., et al. (2004). Characterization and expression analysis of mesenchymal stem cells from human bone marrow and adipose tissue. Cell Physiol. Biochem. 14, 311-324. doi: $10.1159 / 000080341$

Leng, Z., Zhu, R., Hou, W., Feng, Y., Yang, Y., Han, Q., et al. (2020). Transplantation of ACE2- mesenchymal stem cells improves the outcome of patients with COVID-19 Pneumonia. Aging Dis. 11, 216-228. doi: 10.14336/ AD. 2020.0228

Leuning, D. G., Reinders, M. E. J., Li, J., Peired, A. J., Lievers, E., de Boer, H. C., et al. (2017). Clinical-grade isolated human kidney perivascular stromal cells as an organotypic cell source for kidney regenerative medicine. Stem Cells Transl. Med. 6, 405-418. doi: 10.5966/sctm.2016-0053

Levy, O., Kuai, R., Siren, E. M. J., Bhere, D., Milton, Y., Nissar, N., et al. (2020). Shattering barriers toward clinically meaningful MSC therapies. Sci. Adv. 6:eaba6884. doi: 10.1126/sciadv.aba6884

Li, H.-J., Reinhardt, F., Herschman, H. R., and Weinberg, R. A. (2012). Cancerstimulated mesenchymal stem cells create a carcinoma stem cell niche via prostaglandin $\mathrm{E}_{2}$ signaling. Cancer Discov. 2, 840-855. doi: 10.1158/2159-8290. CD-12-0101

Li, X., Zeng, X., Xu, Y., Wang, B., Zhao, Y., Lai, X., et al. (2020). Mechanisms and rejuvenation strategies for aged hematopoietic stem cells. J. Hematol. Oncol. 13:31. doi: 10.1186/s13045-020-00864-8

Li, X., Zhang, Y., Yeung, S. C., Liang, Y., Liang, X., Ding, Y., et al. (2014). Mitochondrial transfer of induced pluripotent stem cell-derived mesenchymal stem cells to airway epithelial cells attenuates cigarette smoke-induced damage. Am. J. Respir. Cell Mol. Biol. 51, 455-465. doi: 10.1165/rcmb.2013-0529OC

Lian, Q., Zhang, Y., Zhang, J., Zhang, H. K., Wu, X., Zhang, Y., et al. (2010). Functional mesenchymal stem cells derived from human induced pluripotent stem cells attenuate limb ischemia in mice. Circulation 121, 1113-1123. doi: 10.1161/CIRCULATIONAHA.109.898312

Lipsitz, Y. Y., Timmins, N. E., and Zandstra, P. W. (2016). Quality cell therapy manufacturing by design. Nat. Biotechnol. 34, 393-400. doi: 10.1038/nbt.3525

Liu, X., Xiang, Q., Xu, F., Huang, J., Yu, N., Zhang, Q., et al. (2019). Single-cell RNA-seq of cultured human adipose-derived mesenchymal stem cells. Sci. Data 6:190031. doi: 10.1038/sdata.2019.31

Lotfy, A., Salama, M., Zahran, F., Jones, E., Badawy, A., and Sobh, M. (2014). Characterization of mesenchymal stem cells derived from rat bone marrow 
and adipose tissue: a comparative study. Int. J. Stem Cells 7, 135-142. doi: 10.15283/ijsc.2014.7.2.135

Mack, A. A., Kroboth, S., Rajesh, D., and Wang, W. B. (2011). Generation of induced pluripotent stem cells from CD34+ cells across blood drawn from multiple donors with non-integrating episomal vectors. PLoS One 6:e27956. doi: 10.1371/journal.pone.0027956

MacQueen, L., Sun, Y., and Simmons, C. A. (2013). Mesenchymal stem cell mechanobiology and emerging experimental platforms. J. R. Soc. Interface 10:20130179. doi: 10.1098/rsif.2013.0179

Madsen, S. D., Russell, K. C., Tucker, H. A., Glowacki, J., Bunnell, B. A., and O'Connor, K. C. (2017). Decoy TRAIL receptor CD264: a cell surface marker of cellular aging for human bone marrow-derived mesenchymal stem cells. Stem Cell Res. Ther. 8:201. doi: 10.1186/s13287-017-0649-4

Marks, P. W., Witten, C. M., and Califf, R. M. (2017). Clarifying stem-cell therapy's benefits and risks. N. Engl. J. Med. 376, 1007-1009. doi: 10.1056/ NEJMp1613723

Maximow, A. A. (1924). Relation of blood cells to connective tissues and endothelium. Physiol. Rev. 4, 533-563. doi: 10.1152/physrev.1924.4.4.533

McLeod, C. M., and Mauck, R. L. (2017). On the origin and impact of mesenchymal stem cell heterogeneity: new insights and emerging tools for single cell analysis. Eur. Cell Mater. 34, 217-231. doi: 10.22203/eCM.v034a14

Moskalev, A. A., Shaposhnikov, M. V., Plyusnina, E. N., Zhavoronkov, A., Budovsky, A., Yanai, H., et al. (2013). The role of DNA damage and repair in aging through the prism of Koch-like criteria. Ageing Res. Rev. 12, 661-684. doi: 10.1016/j.arr.2012.02.001

Muraglia, A., Cancedda, R., and Quarto, R. (2000). Clonal mesenchymal progenitors from human bone marrow differentiate in vitro according to a hierarchical model. J. Cell Sci. 113(Pt 7), 1161-1166.

O'Cearbhaill, E. D., Ng, K. S., and Karp, J. M. (2014). Emerging medical devices for minimally invasive cell therapy. Mayo Clin. Proc. 89, 259-273. doi: 10.1016/j. mayocp.2013.10.020

O'Connor, K. C. (2019). Molecular profiles of Cell-to-Cell variation in the regenerative potential of mesenchymal stromal cells. Stem Cells Int. 2019:5924878. doi: 10.1155/2019/5924878

Oreffo, R. O., Bord, S., and Triffitt, J. T. (1998). Skeletal progenitor cells and ageing human populations. Clin. Sci. (Lond.) 94, 549-555. doi: 10.1042/cs0940549

Ozay, E. I., Vijayaraghavan, J., Gonzalez-Perez, G., Shanthalingam, S., Sherman, H. L., Garrigan, D. T., et al. (2019). Cymerus ${ }^{\text {TM }}$ iPSC-MSCs significantly prolong survival in a pre-clinical, humanized mouse model of Graft-vs-host disease. Stem Cell Res. 35:101401. doi: 10.1016/j.scr.2019.101401

Palumbo, S., Tsai, T.-L., and Li, W.-J. (2014). Macrophage migration inhibitory factor regulates AKT signaling in hypoxic culture to modulate senescence of human mesenchymal stem cells. Stem Cells Dev. 23, 852-865. doi: 10.1089/scd. 2013.0294

Panés, J., García-Olmo, D., Van Assche, G., Colombel, J. F., Reinisch, W., Baumgart, D. C., et al. (2016). Expanded allogeneic adipose-derived mesenchymal stem cells (Cx601) for complex perianal fistulas in Crohn's disease: a phase 3 randomised, double-blind controlled trial. Lancet 388, 1281-1290. doi: 10.1016/ S0140-6736(16)31203-X

Papaccio, F., Paino, F., Regad, T., Papaccio, G., Desiderio, V., and Tirino, V. (2017) Concise review: cancer cells, cancer stem cells, and mesenchymal stem cells: influence in cancer development: stem cells-cancer stem cells interplay. Stem Cells Transl. Med. 6, 2115-2125. doi: 10.1002/sctm.17-0138

Parekkadan, B., van Poll, D., Megeed, Z., Kobayashi, N., Tilles, A. W., Berthiaume, F., et al. (2007). Immunomodulation of activated hepatic stellate cells by mesenchymal stem cells. Biochem. Biophys. Res. Commun. 363, 247-252. doi: 10.1016/j.bbrc.2007.05.150

Park, G. C., Song, J. S., Park, H.-Y., Shin, S.-C., Jang, J. Y., Lee, J.-C., et al. (2016) Role of fibroblast growth factor-5 on the proliferation of human tonsil-derived mesenchymal stem cells. Stem Cells Dev. 25, 1149-1160. doi: 10.1089/scd.2016. 0061

Phinney, D. G. (2012). Functional heterogeneity of mesenchymal stem cells: implications for cell therapy. J. Cell. Biochem. 113, 2806-2812. doi: 10.1002/jcb. 24166

Phinney, D. G., and Pittenger, M. F. (2017). Concise review: MSC-derived exosomes for Cell-Free therapy. Stem Cells 35, 851-858. doi: 10.1002/stem.2575

Prigione, A., Hossini, A. M., Lichtner, B., Serin, A., Fauler, B., Megges, M., et al. (2011). Mitochondrial-associated cell death mechanisms are reset to an embryonic-like state in aged donor-derived iPS cells harboring chromosomal aberrations. PLoS One 6:e27352. doi: 10.1371/journal.pone.0027352

Rahman, M. S., Wruck, W., Spitzhorn, L.-S., Nguyen, L., Bohndorf, M., Martins, S., et al. (2020). The FGF, TGF $\beta$ and WNT axis modulate Self-renewal of Human SIX2+ urine derived renal progenitor cells. Sci. Rep. 10:739. doi: 10. 1038/s41598-020-57723-2

Reinisch, A., Etchart, N., Thomas, D., Hofmann, N. A., Fruehwirth, M., Sinha, S., et al. (2015). Epigenetic and in vivo comparison of diverse MSC sources reveals an endochondral signature for human hematopoietic niche formation. Blood 125, 249-260. doi: 10.1182/blood-2014-04572255

Reuter, S., Gupta, S. C., Chaturvedi, M. M., and Aggarwal, B. B. (2010). Oxidative stress, inflammation, and cancer: how are they linked? Free Radic. Biol. Med. 49, 1603-1616. doi: 10.1016/j.freeradbiomed.2010. 09.006

Rodríguez-Fuentes, D. E., Fernández-Garza, L. E., Samia-Meza, J. A., BarreraBarrera, S. A., Caplan, A. I., and Barrera-Saldaña, H. A. (2021). Mesenchymal stem cells current clinical applications: a systematic review. Arch. Med. Res. 52, 93-101. doi: 10.1016/j.arcmed.2020.08.006

Roson-Burgo, B., Sanchez-Guijo, F., Del Cañizo, C., and De Las Rivas, J. (2016). Insights into the human mesenchymal stromal/stem cell identity through integrative transcriptomic profiling. BMC Genomics 17:944. doi: 10.1186/ s12864-016-3230-0

Samper Agrelo, I., Schira-Heinen, J., Beyer, F., Groh, J., Bütermann, C., Estrada, V., et al. (2020). Secretome analysis of mesenchymal stem cell factors fostering oligodendroglial differentiation of neural stem cells in vivo. IJMS 21:4350. doi: 10.3390/ijms21124350

Sasportas, L. S., Kasmieh, R., Wakimoto, H., Hingtgen, S., van de Water, J. A. J. M., Mohapatra, G., et al. (2009). Assessment of therapeutic efficacy and fate of engineered human mesenchymal stem cells for cancer therapy. Proc. Natl. Acad. Sci. U.S.A. 106, 4822-4827. doi: 10.1073/pnas.0806647106

Schellenberg, A., Lin, Q., Schüler, H., Koch, C. M., Joussen, S., Denecke, B. et al. (2011). Replicative senescence of mesenchymal stem cells causes DNAmethylation changes which correlate with repressive histone marks. Aging (Albany N.Y.) 3, 873-888. doi: 10.18632/aging.100391

Segers, V. F. M., and Lee, R. T. (2008). Stem-cell therapy for cardiac disease. Nature 451, 937-942. doi: 10.1038/nature06800

Shetty, A. K. (2020). Mesenchymal stem cell infusion shows promise for combating coronavirus (COVID-19)- induced pneumonia. Aging Dis. 11, 462-464. doi: 10.14336/AD.2020.0301

Sheyn, D., Ben-David, S., Shapiro, G., De Mel, S., Bez, M., Ornelas, L., et al. (2016). Human induced pluripotent stem cells differentiate into functional mesenchymal stem cells and repair bone defects. Stem Cells Transl. Med. 5, 1447-1460. doi: 10.5966/sctm.2015-0311

Shibue, T., and Weinberg, R. A. (2017). EMT, CSCs, and drug resistance: the mechanistic link and clinical implications. Nat. Rev. Clin. Oncol. 14, 611-629. doi: 10.1038/nrclinonc.2017.44

Sivasubramaniyan, K., Lehnen, D., Ghazanfari, R., Sobiesiak, M., Harichandan, A., Mortha, E., et al. (2012). Phenotypic and functional heterogeneity of human bone marrow- and amnion-derived MSC subsets. Ann. N. Y. Acad. Sci. 1266, 94-106. doi: 10.1111/j.1749-6632.2012.06551.x

Spitzhorn, L.S., Rahman, M.S., Schwindt, L., Ho, H.T., Wruck, W., Bohndorf M., et al. (2017). Isolation and molecular characterization of amniotic fluidderived mesenchymal stem cells obtained from caesarean sections. Stem Cells Int. 2017:5932706. doi: 10.1155/2017/5932706

Spitzhorn, L.-S., Kordes, C., Megges, M., Sawitza, I., Götze, S., Reichert, D., et al. (2018). Transplanted human pluripotent stem cell-derived mesenchymal stem cells support liver regeneration in gunn rats. Stem Cells Dev. 27, 1702-1714. doi: $10.1089 / \mathrm{scd} .2018 .0010$

Spitzhorn, L.-S., Megges, M., Wruck, W., Rahman, M. S., Otte, J., Degistirici, Ö, et al. (2019). Human iPSC-derived MSCs (iMSCs) from aged individuals acquire a rejuvenation signature. Stem Cell Res. Ther. 10:100. doi: 10.1186/ s13287-019-1209-x

Stolzing, A., and Scutt, A. (2006). Age-related impairment of mesenchymal progenitor cell function. Aging Cell 5, 213-224. doi: 10.1111/j.1474-9726.2006. 00213.x

Strahl, B. D., and Allis, C. D. (2000). The language of covalent histone modifications. Nature 403, 41-45. doi: 10.1038/47412 
Suchanek, J., Soukup, T., Visek, B., Ivancakova, R., Kucerova, L., and Mokry, J. (2009). Dental pulp stem cells and their characterization. Biomed. Pap. Med. Fac. Univ. Palacky Olomouc Czech. Repub. 153, 31-35. doi: 10.5507/bp.2009. 005

Sui, B.-D., Zheng, C.-X., Li, M., Jin, Y., and Hu, C.-H. (2020). Epigenetic regulation of mesenchymal stem cell homeostasis. Trends Cell Biol. 30, 97-116. doi: 10. 1016/j.tcb.2019.11.006

Sukarieh, R., Joseph, R., Leow, S. C., Li, Y., Löffler, M., Aris, I. M., et al. (2014). Molecular pathways reflecting poor intrauterine growth are found in Wharton's jelly-derived mesenchymal stem cells. Hum. Reprod. 29, 2287-2301. doi: 10. 1093/humrep/deu209

Sun, C., Wang, L., Wang, H., Huang, T., Yao, W., Li, J., et al. (2020). Singlecell RNA-seq highlights heterogeneity in human primary Wharton's jelly mesenchymal stem/stromal cells cultured in vitro. Stem Cell Res. Ther. 11:149. doi: 10.1186/s13287-020-01660-4

Tanavde, V., Vaz, C., Rao, M. S., Vemuri, M. C., and Pochampally, R. R. (2015). Research using Mesenchymal Stem/Stromal Cells: quality metric towards developing a reference material. Cytotherapy 17, 1169-1177. doi: 10.1016/j.jcyt. 2015.07.008

Tzouanas, S. N., Ekenseair, A. K., Kasper, F. K., and Mikos, A. G. (2014). Mesenchymal stem cell and gelatin microparticle encapsulation in thermally and chemically gelling injectable hydrogels for tissue engineering. J. Biomed. Mater. Res. A 102, 1222-1230. doi: 10.1002/jbm.a.35093

Vinardell, T., Sheehy, E. J., Buckley, C. T., and Kelly, D. J. (2012). A comparison of the functionality and in vivo phenotypic stability of cartilaginous tissues engineered from different stem cell sources. Tissue Eng. Part A 18, 1161-1170. doi: 10.1089/ten.TEA.2011.0544

Viswanathan, S., Keating, A., Deans, R., Hematti, P., Prockop, D., Stroncek, D. F., et al. (2014). Soliciting strategies for developing cell-based reference materials to advance mesenchymal stromal cell research and clinical translation. Stem Cells Dev. 23, 1157-1167. doi: 10.1089/scd.2013.0591

Viswanathan, S., Shi, Y., Galipeau, J., Krampera, M., Leblanc, K., Martin, I., et al. (2019). Mesenchymal stem versus stromal cells: international society for cell \& gene therapy (ISCT ${ }^{\circledR}$ ) mesenchymal stromal cell committee position statement on nomenclature. Cytotherapy 21, 1019-1024. doi: 10.1016/j.jcyt.2019.08.002

von Bahr, L., Sundberg, B., Lönnies, L., Sander, B., Karbach, H., Hägglund, H., et al. (2012). Long-term complications, immunologic effects, and role of passage for outcome in mesenchymal stromal cell therapy. Biol. Blood Marrow Transplant. 18, 557-564. doi: 10.1016/j.bbmt.2011.07.023

Wagner, W., Bork, S., Horn, P., Krunic, D., Walenda, T., Diehlmann, A., et al. (2009). Aging and replicative senescence have related effects on human stem and progenitor cells. PLoS One 4:e5846. doi: 10.1371/journal.pone.0005846

Wagner, W., Horn, P., Castoldi, M., Diehlmann, A., Bork, S., Saffrich, R., et al. (2008). Replicative senescence of mesenchymal stem cells: a continuous and organized process. PLoS One 3:e2213. doi: 10.1371/journal.pone.0002213

Wagner, W., Wein, F., Seckinger, A., Frankhauser, M., Wirkner, U., Krause, U., et al. (2005). Comparative characteristics of mesenchymal stem cells from human bone marrow, adipose tissue, and umbilical cord blood. Exp. Hematol. 33, 1402-1416. doi: 10.1016/j.exphem.2005.07.003

Wang, L., Niu, N., Li, L., Shao, R., Ouyang, H., and Zou, W. (2018). H3K36 trimethylation mediated by SETD2 regulates the fate of bone marrow mesenchymal stem cells. PLoS Biol. 16:e2006522. doi: 10.1371/journal.pbio. 2006522

Wang, L.-T., Ting, C.-H., Yen, M.-L., Liu, K.-J., Sytwu, H.-K., Wu, K. K., et al. (2016). Human mesenchymal stem cells (MSCs) for treatment towards immune- and inflammation-mediated diseases: review of current clinical trials. J. Biomed. Sci. 23:76. doi: 10.1186/s12929-016-0289-5

Wang, X., Kimbrel, E. A., Ijichi, K., Paul, D., Lazorchak, A. S., Chu, J., et al. (2014). Human ESC-derived MSCs outperform bone marrow MSCs in the treatment of an EAE model of multiple sclerosis. Stem Cell Rep. 3, 115-130. doi: 10.1016/j.stemcr.2014.04.020

Warnes, G. R., Bolker, B., Bonebakker, L., Gentleman, R., Liaw, W. H. A., Lumley, T., et al. (2015). gplots: Various R Programming Tools for Plotting Data. Available online at: http://CRAN.R-project.org/package=gplots (accessed Nov 11, 2020).

Wickham, H. (2009). Ggplot2: Elegant Graphics for Data Analysis. New York, NY: Springer.

Wilson, A., Webster, A., and Genever, P. (2019). Nomenclature and heterogeneity: consequences for the use of mesenchymal stem cells in regenerative medicine. Regen. Med. 14, 595-611. doi: 10.2217/rme-2018-0145
Wolmarans, E., Mellet, J., Durandt, C., Joubert, F., and Pepper, M. S. (2021). SingleCell transcriptome analysis of human adipose-derived stromal cells identifies a contractile cell subpopulation. Stem Cells Int. 2021:5595172. doi: 10.1155/2021/ 5595172

Wruck, W., Graffmann, N., Kawala, M.-A., and Adjaye, J. (2017). Concise review: current status and future directions on research related to nonalcoholic fatty liver disease. Stem Cells 35, 89-96. doi: 10.1002/stem.2454

Xin, Y., Wang, Y., Zhang, H., Li, J., Wang, W., Wei, Y., et al. (2010). Aging adversely impacts biological properties of human bone marrow-derived mesenchymal stem cells: implications for tissue engineering heart valve construction. Artif. Organs 34, 215-222. doi: 10.1111/j.1525-1594.2009.00824.x

Xu, L., Liu, Y., Sun, Y., Wang, B., Xiong, Y., Lin, W., et al. (2017). Tissue source determines the differentiation potentials of mesenchymal stem cells: a comparative study of human mesenchymal stem cells from bone marrow and adipose tissue. Stem Cell Res. Ther. 8:275. doi: 10.1186/s13287-017-0716- $\mathrm{x}$

Yang, Y., Ye, G., Zhang, Y.-L., He, H.-W., Yu, B.-Q., Hong, Y.-M., et al. (2020). Transfer of mitochondria from mesenchymal stem cells derived from induced pluripotent stem cells attenuates hypoxia-ischemia-induced mitochondrial dysfunction in PC12 cells. Neural Regen. Res. 15, 464-472. doi: 10.4103/16735374.266058

Yang, Y.-H. K., Ogando, C. R., Wang See, C., Chang, T.-Y., and Barabino, G. A. (2018). Changes in phenotype and differentiation potential of human mesenchymal stem cells aging in vitro. Stem Cell Res. Ther. 9:131. doi: 10.1186/ s13287-018-0876-3

Yin, B., Yu, F., Wang, C., Li, B., Liu, M., and Ye, L. (2019). Epigenetic control of mesenchymal stem cell fate decision via histone methyltransferase Ash1l. Stem Cells 37, 115-127. doi: 10.1002/stem.2918

Zha, K., Li, X., Yang, Z., Tian, G., Sun, Z., Sui, X., et al. (2021). Heterogeneity of mesenchymal stem cells in cartilage regeneration: from characterization to application. NPJ Regen. Med. 6:14. doi: 10.1038/s41536-021-00 $122-6$

Zhang, J., Muise, E. S., Han, S., Kutchukian, P. S., Costet, P., Zhu, Y., et al. (2020). Molecular profiling reveals a common metabolic signature of tissue fibrosis. Cell Rep. Med. 1:100056. doi: 10.1016/j.xcrm.2020.100056

Zhang, Y., Yu, Z., Jiang, D., Liang, X., Liao, S., Zhang, Z., et al. (2016). iPSCMSCs with high intrinsic MIRO1 and sensitivity to TNF- $\alpha$ yield efficacious mitochondrial transfer to rescue anthracycline-induced cardiomyopathy. Stem Cell Rep. 7, 749-763. doi: 10.1016/j.stemcr.2016.08.009

Zhao, C., and Ikeya, M. (2018). Generation and applications of induced pluripotent stem cell-derived mesenchymal stem cells. Stem Cells Int. 2018:9601623. doi: $10.1155 / 2018 / 9601623$

Zhou, S., Greenberger, J. S., Epperly, M. W., Goff, J. P., Adler, C., Leboff, M. S., et al. (2008). Age-related intrinsic changes in human bone-marrow-derived mesenchymal stem cells and their differentiation to osteoblasts. Aging Cell 7, 335-343. doi: 10.1111/j.1474-9726.2008.00377.x

Zhou, T., Yuan, Z., Weng, J., Pei, D., Du, X., He, C., et al. (2021). Challenges and advances in clinical applications of mesenchymal stromal cells. J. Hematol. Oncol. 14:24. doi: 10.1186/s13045-021-01037-x

Zhou, X., Hong, Y., Zhang, H., and Li, X. (2020). Mesenchymal Stem cell senescence and rejuvenation: current status and challenges. Front. Cell Dev. Biol. 8:364. doi: 10.3389/fcell.2020.00364

Conflict of Interest: The authors declare that the research was conducted in the absence of any commercial or financial relationships that could be construed as a potential conflict of interest.

Publisher's Note: All claims expressed in this article are solely those of the authors and do not necessarily represent those of their affiliated organizations, or those of the publisher, the editors and the reviewers. Any product that may be evaluated in this article, or claim that may be made by its manufacturer, is not guaranteed or endorsed by the publisher.

Copyright (ㄷ 2021 Wruck, Graffmann, Spitzhorn and Adjaye. This is an openaccess article distributed under the terms of the Creative Commons Attribution License (CC BY). The use, distribution or reproduction in other forums is permitted, provided the original author(s) and the copyright owner(s) are credited and that the original publication in this journal is cited, in accordance with accepted academic practice. No use, distribution or reproduction is permitted which does not comply with these terms. 